\title{
Short term electrostimulation enhancing neural repair in vitro using large charge capacity nanostructured electrodes
}

\author{
M. P. Lichtenstein ${ }^{\dagger+\#}$, E. Pérez ${ }^{\dagger \#}$, L. Ballesteros ${ }^{\dagger}$, C. Suñol $^{\dagger \dagger}$, N. Casañ-Pastor ${ }^{\dagger \neq *}$ \\ † Institut Ciència de Materials Barcelona (ICMAB, CSIC), Campus UAB, E-08193, Bellaterra, Barcelona, Spain . * Email: \\ nieves@icmab.es \\ ${ }^{+\dagger}$ Institut d'Investigacions Biomèdiques de Barcelona (IIBB, CSIC); Institut d'Investigacions Biomèdiques August Pi i \\ Sunyer (IDIBAPS). c/Rosselló 161, 08036 Barcelona, Spain. " CIBER Epidemiología y Salud Pública (CIBERESP), Spain \\ \#\#Equal contribution
}

Keywords: Electrostimulation, electrode materials, charge capacity, in vitro neurite regeneration, iridium oxide, graphene, conducting polymers

\begin{abstract}
Electrostimulation of the neural system in functional or repair therapies require new materials that protect the living system from electric field (EF) effects at the interface. Intercalation materials offer an alternative to radical formation during stimulation. Furthermore, nanostructuring of the electroactive material used as electrode offers an enlargement of the charge capacity which in turn involves changes in the EF effect. In this work, electric field stimulation of cortical neuron cultures has been applied in an in vitro model of lesion, namely, a physical scratch in the cell culture creates a cell-free area reminiscent of a lesion where new neurites grow. Regeneration of the "wound" zone upon EF stimulation is observed for various types of electrode materials, and compared to the spontaneous process and to platinum electrodes. Significantly, electric field effects are highly dependent on the electrode material used, even for the same charge delivered and similar impedance values. Electrode coatings with large charge storage capacity yield significantly better results than bare Pt electrodes. Neurite outgrowth at the scratched "wound" zone is lowest, below spontaneous regeneration, when using Pt electrodes. On the other hand, electroactive materials, such as bilayers of PEDOT and polypyrrole with Lysine counterions or iridium oxide-pristine graphene hybrids, promote further regeneration. Beyond impedance considerations, the optimal material is the nanostructured one with the largest charge capacity, even at low charge deliveries. It is remarkable that IrOxgraphene hybrids reach regenerations above spontaneous case in very short stimulation times, for equal charge deliveries and potential protocols. The implications from the results suggest that EF application using these new coatings, may have an immediate use in safer electrostimulation procedures, and open routes for much needed neural repair.
\end{abstract}




\section{Introduction}

The existence of endogenous electric fields in living organisms is at the core of life mechanisms and of spontaneous self repair. External fields could interact therefore with endogenous fields suggesting possible actions on to enhance healing, regeneration, growth and transmission of electrical signals. [1,2] To date, functional electrical stimulation is used clinically to alleviate symptoms of pathologies such as Parkinson disease, depression and epilepsy. $[3,4]$ Clinical procedures exist on functional electrostimulation in which EF application alleviates a non desirable behavior in neurons or in neural communication. $[5,6]$ Electrodes usually implanted are based on purely capacitive materials such as Pt and its alloys, TiN etc, for which the EF charge delivered easily overcomes the capacitance value and yields faradaic processes such as $\mathrm{O}_{2}$ reduction and $\mathrm{H}_{2} \mathrm{O}$ oxidation and the associated radical oxygen species at the interface. [4] In order to minimize this side effect, AC pulses are applied with net charge near zero, in cases of functional stimulation, but short lived radicals favor an inflammatory response that yields to encapsulation of the material by the surrounding tissue and the final removal of the electrode is necessary. [7] Alternatively, using a repair electrostimulation approach, it has been proven that neural cells respond to external direct fields (DC) as they do to endogenous electric fields (EF), $[1,8]$ but if a traumatic lesion occurs, scar tissue is formed inhibiting regeneration. Before the formation of the scar, there is an optimal time for external therapies to be applied (chemical, electrical) that could aid to enhance the spontaneous mechanisms that favor repair. [9] Repair in traumatic lesions is far away in the horizon, and nonetheless a general consensus exists on the need of new materials for implanted electrodes to be inert while protecting the tissue, before that objective is achieved. Closely related fields like bionic implants, sensor and recording devices that require optimal interfacing share the same needs and materials, and benefit from findings in the electrostimulation area.

Individual Xenopus neurons in vitro have shown a statistical migration (60 \%) upon EF DC stimulation, and also dendrite directioning towards the cathode. However axons do not show such effect. [1,2,10-12] Surprisingly, other types of cells migrate towards the anode, with no interpretation yet available on that difference. In these cases, the external field applied $(\approx 100$ $\mathrm{mV} / \mathrm{mm}$ ) is only a perturbing field that does not affect membrane permeability, and is much lower than the DC fields used for electroporation for example, or for membrane depolarizing. $[1,9]$ Typically, all these experiments are with electrodes external to the cell culture because of the lack of inert electrodes for $\mathrm{DC}$ experiments $(\mathrm{Ag} / \mathrm{AgCl}$ electrodes in the previous case). On the other hand, in vivo injuries, containing bundles of neurons, are known to generate additional injury potentials that persist during some time after the trauma, and that are relevant in wound healing. In those cases, in vivo assays evidence some sensory improvement after EF stimulation of the central nervous system (CNS) using external electrodes. [13]

As compared with chemically inert Pt electrodes, electroactive materials with mixed valence states are able to undergo redox intercalation reactions themselves, and offer a safe alternative pathway for the faradaic exchange at the interface derived from radical formation, 
and thus offer an outstandinng possibility for implanted electrodes. Electrodeposited Iridium oxide (IrOx), [14-19] conducting polymers based on polypyrrole (PPy) or 3,4polyethylenedyoxithiophene (PEDOT), [20-23] polymers hybridized with nanocarbons [24,25] and the new hybrids of IrOx with nanocarbons, [26-28] undergo a redox change that is translated in a large charge storage capacity for the electrode. The capacitive part thus remains as a small contribution vs the pseudocapacitor or coulombic internal faradaic part. The electrode charge storage capacity (CSC) available from these intercalation processes is a measure of the extent in which the electrode may be used in the DC mode electric field, as it happens in batteries. Electric field application using intercalation materials (or coatings of them) with large charge capacities (CSC) is expected to have distinct effects since that internal redox change is an alternative to radical formation, and it must allow working in the lower EF frequency range with larger charge delivery in DC mode and within CSC limits. This work is an in vitro proof of that concept. There is a wide span of CSC values that have been recently achieved, much larger than capacitive values in platinum, its alloys or TiN. [29] Thus, in previous works we have shown that it is possible to move from the CSC $100 \mu \mathrm{C} / \mathrm{cm}^{2}$ range for Pt electrodes to $5 \mathrm{mC} / \mathrm{cm}^{2}$ in electrodeposited IrOx and $20 \mathrm{mC} / \mathrm{cm}^{2}$ if deposition is made dynamically for IrOx. [18] However the more dramatic improvement in CSC, $120 \mathrm{mC} / \mathrm{cm}^{2}$, is achieved for graphene or carbon nanotubes nanostructured with IrOx, forming IrOx-nanocarbons hybrids. [26-28] Nanostructuring of conducting polymers, however, yields CSC that remain in the range of 2 to $20 \mathrm{mc} / \mathrm{cm}^{2}$. [30] Furthermore, the contribution of nanocarbons to charge capacities has been shown to be remarkable when large stability is required, as compared to macro carbons as graphite. $[27,28]$

The chemistry effects of the substrate material used in neural cell adhesion, has been also shown to be significant in cultures without EF stimulation. Neuronal viability results evidence that cells distinguish the actual surface chemistry, well above topographical cues $[31,32]$ of the material, while primary neuron viability is compromised when in the vicinity of some materials like PEDOT-PSS. [30] DC electric fields stimulation using conducting polymers such as polypyrrole and PEDOT-PSS coatings have also reported an increased neurite outgrowth during the differentiation of the PC12 cell line derived from rat adrenal medulla, [21,33] which may not have the same behavior as primary neurons. We have proven that aminoacid-doped polymer bilayers PEDOT-polypyrrole composites, are superior in primary neuronal cell viability studies with respect to the PEDOT or polypyrrole -PSS counterparts, while maintaining significant charge capacities. [30] Carbon materials, [21,34] IrOx and IrOx-nanocarbon hybrid materials show good compatibility with neuronal cells preserving full functional properties. [18,26-28,35] IrOx has also been tested usually in $A C$ electrostimulation experiments in the frequency range used for Parkinson symptoms relief, $[15,16,19,36]$ based on the impedance decrease when this oxide is used as coating. Furthermore, electrostimulation of neurons has been achieved by using nanocarbon electrodes offering a significant neural interface in recording, stimulation and bionics. [34,37] Likewise, chemical-induced depolarizing stimulus evokes action potentials eventually releasing the neurotransmitters glutamate and GABA in cortical neurons grown on IrOx-nanocarbon electrodes evidencing the optimal function of neurons on those materials. $[26,28]$ More recently neurite growth has been observed in graphene based surfaces, [38] CNT 
[39-41] and in GO 3D fibers, [42] confirming that graphene oxide and CNT are permissive substrates for neuronal cell growth. Systems that contain pristine graphene have also been studied, $[28,43]$ and result optimal as a substrate for neural growth over long periods of time, well above graphene oxide, [43] with clear evidence that defects in graphene alter neural compatibility. However, no previous use of large capacity electrodes is reported.

While electric field application in cells have been mainly studied for its effect on cell migration and neurite elongation using external electrodes, the effects of different types of inner implanted electrodes on a specific in vitro model of wounds needs study. $[5,44]$ In this direction, this work attempts to show the significant effect of new type of nanostructured mixed valence materials with intercalation properties and large charge storage capacity (CSC) vs capacitive Pt. Effects of low frequency electrostimulation experiments with net DC charge delivered in both neuronal viability and neurite regeneration are studied. In order to do so, an in vitro model simulating a neuronal lesion has been designed and characterized, creating an area free of cells and neurites reminiscent of a wound, and used for the first time in presence of EF. After wounding, neurites sprout out and start to grow perpendicularly respect the direction of the wound, spontaneously. The regeneration of neurites into the cell free area was studied in the presence or absence of EF, and different materials (either platinum electrodes or the new generation of nanostructured materials) with common intercalation properties but different capacities [26-28,30] were used as electrodes.

The significance of the work lies in the use of new materials with large CSC as implanted electrodes in EF stimulation and also in the comparison of the implanted electrodes with similar intercalation mechanisms but different charge capacities and chemistry. It also lies on the in vitro wound model proposed and used for the first time in EF studies. Also, new EF protocols are used that allow homogeneity of the electric field and differentiation of anode and cathode zones with control of delivered charge to allow comparison among electrodes. The results show that the new nanostructured materials enhance spontaneous regeneration, while Pt electrodes decrease it. This work shows a step ahead in materials for electrostimulation, thanks to the combination of an in vitro model for a lesion, a scratch scheme at the cell culture, [45] with electrostimulation. Using the same charge delivered and very short stimulation times, the effect of EF depends largely on the specific material used and its electrochemical properties. Intercalation mixed valence materials, bilayer polymer or IrOx-graphene hybrids work better than platinum for the same charge delivered, and when both electrodes are coated with the same material, with nanostructured IrOx-graphene being the best option.

\section{Materials and Methods}

Coatings of various materials are prepared by electrodeposition on Pt coated glass, according to previously described procedures, $[18,28,30,46]$ and optimizing thicknesses for transparency during cell culture studies: Microscope glass slides with dimensions $24 \times 70 \mathrm{~mm}$, are 
deposited with Ti $(5 \mathrm{~nm})$ and Pt $(12 \mathrm{~nm})$ by thermal evaporation in the two border zones with dimensions $20 \times 20 \mathrm{~mm}$ (See below in Figure 4), leaving two non plated spaces at the center and near each platinum side. Cell cultures over borosilicateH are placed on those non conducting parts which become respectively the anode and cathode sides of the final electrochemical cell, and they will be separated by a salt bridge in the final device. Alternatively, $2 \mathrm{~cm}$ wide Pt ribbons with $L$ shape were coated and used vertically in each side of the electrochemical cell. Results are identical and only the glass coated electrodes will be shown. All intercalation materials have been tested before by Electrochemical Quartz Microbalance $[18,30]$ to intercalate $\mathrm{Na}^{+}$ions in the media used, in molar ratios defined by the applied charge. All materials will be used in this paper using the same charge delivered for correct comparison. All, with exception of Pt, produce therefore the same local change at the interface in terms of $\mathrm{Na}^{+}$concentration.

Electrodeposition of coatings in each Pt side was controlled using a potentiostat/galvanostat (Bio-logic Science Instruments, model VMP3). We used a threeelectrode cell configuration containing $60 \mathrm{~mL}$ electrolyte solution, with a Pt foil ( $10 \mathrm{~cm}^{2}$ exposure) as counter electrode and Pt wire $\left(0.5 \mathrm{~mm}\right.$ diameter, $78.54 \mathrm{~mm}^{2}$ exposure) as quasi-reference electrode. The pseudoreference platinum has shown previously to be stable vs $\mathrm{Ag} / \mathrm{AgCl}$ during $\mathrm{CV}$, possibly due to the formation of an oxide on the surface, and has been used successfully as such, $[47,48]$ while preventing insulating effects in the electric field force lines. The working electrode, where coatings were deposited, was the Pt coated soda lime glass, or bulk Pt ribbon. Given the sensitivity of neurons to the nearby environment, electrode coatings reported in this work are chosen from previous neural viability studies performed on cortical neurons cultures on poly-L-Lysine coated surfaces (See Supplementary Material Table S1 and Figure S1 and ref. $[18,26-28,30,46]$ and note that PEDOT-PSS do not support cell growth at 4DIV). In addition, among the best cellular response materials, a range of charge storage capacities and material types have been selected. The chosen materials represent the best examples of two types, IrOxpristine graphene (IrOx-eG) as example of IrOx hybrids with nanocarbons, and PEDOTpolypyrrole-Lysine bilayer (B-Lys) as a conducting polymer. In that way, we cover a wide span of charge storage capacities, CSC. Both have been compared with uncoated platinum electrodes which are used in present clinical applications. [29] Additional comparisons are also shown with IrOx, and other polymers when possible, to help place some of the properties in context.

PEDOT-polypyrrole-Lysine bilayers and PEDOT-PSS coatings are obtained by electrodeposition on the Pt-coated glass as described before. $[30,46]$ The formation of the upper layer of polypyrrole-lysine has proven before to yield better neural viability, while polypyrrolelysine gains stability when grown on PEDOT instead of Pt. Pyrrole (Sigma-Aldrich 98\%), was vacuum-distilled and then stored at $-10 \stackrel{\circ}{ } \mathrm{C}$ until use. EDOT (3,4-ethylenedyoxithiophene) (SigmaAldrich) was purchased and stored at 5 ㅇ $\mathrm{C}$ until use. Pyrrole and EDOT at concentrations $0.1 \mathrm{M}$ and $0.01 \mathrm{M}$ respectively were dissolved in $\mathrm{pH} 7.4$ phosphate buffer solution (PB) (PB is $\mathrm{NaH}_{2} \mathrm{PO}_{4}$ and $\mathrm{Na}_{2} \mathrm{HPO}_{4}, 0.1 \mathrm{M}$ total concentration) in order to keep a constant $\mathrm{pH}$, and were deposited sequencially, polypyrrole over previously deposited PEDOT, both with L-Lysine counterion (97\% Aldrich) using a potendiodynamic method from rest potential $\left(E_{o}\right)$ to $0.9 \mathrm{~V} v s$ Pt at $5 \mathrm{mV} / \mathrm{s}$ up to a 
desired charge of $64.8 \mathrm{mC} / \mathrm{cm}^{2}$ for PEDOT, and the same dynamic procedure from $\mathrm{E}_{\mathrm{o}}$ to $0.8 \mathrm{~V} v \mathrm{~s}$ Pt at a speed of $10 \mathrm{mV} / \mathrm{s}$ up to a desired charge of $48.4 \mathrm{mC} / \mathrm{cm} 2$ for PPy. PEDOT-PSS prepared as described [46] from 0.1 M EDOT solutions and $0.1 \mathrm{M}$ poly(sodium 4-styrene sulfonate) (PSS) (Aldrich) and potentiostatic oxidation at $0.9 \mathrm{~V}$ vs Pt up to a total charge delivery of $90 \mathrm{mC} / \mathrm{cm}^{2}$. Lysine aminoacid diffusion in aqueous media was determined in films that were exposed to similar conditions to cell cultures, and resulted negative.

IrOx-pristine graphene hybrid coatings (IrOx-eG) were obtained from solutions of exfoliated graphene and $\mathrm{IrCl}_{3}$ precursor as previously described. [28] Graphene suspensions were prepared by electrochemical exfoliation [28] in $0.02 \mathrm{M} \mathrm{H}_{2} \mathrm{C}_{2} \mathrm{O}_{4}$ (Aldrich, $99 \%$ ) solutions of a graphite rod (Goodfellow, $99.997 \%, 5.0 \mathrm{~mm}$ diameter, used as anode in a two electrode configuration with platinum wire (Goodfellow, 99.9\%, $0.5 \mathrm{~mm}$ diameter) as cathode. Constant current, $39 \mathrm{~mA} / \mathrm{cm}^{2}$, was applied for $5 \mathrm{~h}$. The resulting black graphene suspension was then sonicated $1 \mathrm{~h}$, being stable during long periods of time. Then, $0.004 \mathrm{M}$ iridium solutions were obtained by addition of $\operatorname{IrCl}_{3} \cdot \mathrm{H}_{2} \mathrm{O}$ (Aldrich, $99.9 \%$ ) to the described graphene solutions and 30 minutes sonication. The final $\mathrm{pH}=11$ was adjusted by slow addition of $\mathrm{K}_{2} \mathrm{CO}_{3}$ (Aldrich, 99\%) saturated solution, and the solution aged 7 days at $37 \stackrel{\circ}{\circ}$ and stored at 4 으 $\mathrm{C}$ until use. At this stage, IrOx clusters described previously [18] are attached to graphene platelets in suspension. IrOx-eG hybrids were obtained as coatings by dynamic anodic electrodeposition on previously described Pt coated glass substrates from IrOx-eG solutions, using a three-electrode electrochemical cell. A Pt sheet (Goodfellow, $99.9 \%$ ) of equal area was used as counterelectrode, and positioned parallel (1 cm separation). A $0.5 \mathrm{~mm}$ diameter Pt wire (Goodfellow, $99.9 \%$ ) was used as pseudo-reference electrode, $\mathrm{E}$ vs $\mathrm{Ag} / \mathrm{AgCl} 0.02 \mathrm{~V}$. Coatings were obtained by 10-50 cycles potentiodynamic sweeps at $5 \mathrm{mV} / \mathrm{s}$, from open circuit potential (OPC) (near $0.0 \mathrm{~V}$ ) to $+0.70 \mathrm{~V}$ vs Pt. Reference pure IrOx coatings were synthesized similarly without the graphene solution, according to reference. [18] The presence of graphene prevents the self limiting effect observed for pure IrOx, yielding a larger charge capacity electrode (see below).

Characterization of the coatings was performed by various techniques as described in previous references, $[18,26-28,30,46]$ and the results showed the full reproducibility of the coatings. For the specific thickness of the coatings used in this work, as calibrated from charge used, cyclic voltammetries were run and CSC was recalculated, using the same speed in all cases, $10 \mathrm{mV} / \mathrm{s}$. Electrochemical Impedance Spectroscopy (EIS) measurements at room temperature were also performed on samples with the same electrode geometrical area $(10 \times 10 \mathrm{~mm})$ in phosphate buffered saline solution (PBS) (PBS, in $\mathrm{mM}: 137 \mathrm{NaCl}, 2.7 \mathrm{KCl}, 10 \mathrm{Na}_{2} \mathrm{HPO}_{4}, 1.8 \mathrm{KH}_{2} \mathrm{PO}_{4}$ at $\mathrm{pH}$ 7.4). Both were carried out using a Biologic Potentiostat, and a three electrode configuration. The working electrode is the Pt coated with the material under study, the counterelectrode is a Pt rectangular piece with the same area but two conducting sides, while an $\mathrm{Ag} / \mathrm{AgCl}(\mathrm{KCl}$ sat.) reference electrode is used for EIS and a pseudoreference Pt wire electrode for $\mathrm{CV}$. For EIS, a sinusoidal AC wave of $10 \mathrm{mV}$ amplitude is used at open circuit potential (vs $\mathrm{Ag} / \mathrm{AgCl}$ ) and measurements made in the $100 \mathrm{mHz}-100 \mathrm{KHz}$ frequency range. Other coatings where a previous 4 DIV cell culture were not optimal were discarded (A summary of them is 
supplied in Supplementary Material Table S1 (ex. PEDOT single layers, etc). Electrochemical Quartz microbalance and XPS studies had been published by the authors in previous works demonstrating hydrated $\mathrm{Na}^{+}$intercalation during reduction in cell culture media for both materials shown here as electrodes. [18,30] (See also scheme 1 )

EF electrostimulation device was set up using a hollow plastic holder Nunc Thermo Fisher Scientific (cell culture tested) on top of the glass and sealed in the external part with fish tank silicone, so that no liquid leaks out. Once the device was set, its tightness was checked during 5 days in PBS solution and at 37 ㅇ․ The electrodes and device were sterilized by two hourimmersion in distilled water at $60 \stackrel{\circ}{ } \mathrm{C}$. Thereafter, two poly-L-Lysine-coated borosilicate pieces containing cortical neurons that have been cultured for 5 days in vitro (DIV) were placed in the central zones of the glass slide, close to the electrodes.

Cell cultures in which an electric field was applied were compared to cases in which no field is applied, to assure electric field homogeneity and set up design including the saline bridge. After optimizing the set up, (homogeneous electric fields are achieved as shown by cell cultures homogeneity), an in vitro model simulating an injury, is applied. For that, a scratch is made in the previously stabilized cell culture after 5 DIV and neurite regeneration is studied in absence and in presence of electric fields, both in the anode and in the cathode sides of the electrochemical device. Therefore, four types of assays are performed: a) cell cultures with no scratch and no electric field, b) cultures with scratch and no electric field applied, c) cultures with no scratch and applied electric field and d) cultures with scratch and electric field.

Neurons were obtained from the cerebral cortex of 16 day-old NMRI mice embryos (Charles River). The embryos cells are mixed disregarding their sex. Primary neuronal cultures were prepared as previously described. [28,30] Briefly, after dissection and removal of meninges, tissue was minced and dissociated in KREBS buffer containing $\mathrm{MgSO}_{4}$, albumin, trypsin and DNAase. The resulting cell suspension was re-suspended in DMEM plus $10 \%$ fetal bovine serum and seeded at a density 80000 cells $/ \mathrm{cm}^{2}$ for viability assays and 160000 cells $/ \mathrm{cm}^{2}$ for neurite regeneration in the scratch assay. After two hours the medium is substituted by Neurobasal medium supplemented with L-glutamine, B27 supplement, Penicillin-Streptomycin. The replacement of the plating medium with serum-free Neurobasal was intended to prevent glial growth. Cells were grown on top of poly-L-Lysine pre-coated borosilicateH glass $(22 \mathrm{~mm}$ diameter) for at least 5 DIV. Poly-L-Lysine had been coated previously from incubation of borosilicate in $45 \mu \mathrm{g} / \mathrm{mL}$ poly-L-Lysine solutions at $37 \stackrel{\circ}{\circ}$. Animals were handled in compliance with protocols approved by the Generalitat de Catalunya, Spain, following European Union Guidelines.

Inmunocitochemistry for neurite outgrowth and cell quantification: Neurobasal medium was removed from the culture wells, and neurons were fixed with $4 \%$ paraformaldehide at room temperature for $10 \mathrm{~min}$. After washing with phosphate buffered saline (PBS), cells were permeabilized with PBS containing $0.15 \%$ Triton X-100 for 15 min, and blocked with $1 \%$ bovine serum albumin (BSA) for 1 hour to prevent unspecific binding. Primary antibody against Tau 
(Sigma T-6402), which is a specific component of neuronal cytoskeleton, was diluted 1:500 in PBS-BSA $0.1 \%$ and incubated overnight on cells. On the following day, and after three washes with PBS, Alexa-488 anti-rabbit (Molecular Probes, 1:1000) was used as secondary antibody to enable green fluorescence visualization. Bis-benzimide (Sigma, $5 \mu \mathrm{M}$ for 5 minutes) was used for nuclear staining and identification of living cells. Photographs were taken using a Leica DMI 4000B fluorescence microscope. Fluorescent images of both nuclear and Tau staining were combined and used for quantification of healthy neurons on materials. Six radial fields ( $563 \times 401$ $\mu \mathrm{m}$ each) were systematically photographed in each sample and cell counts were extrapolated to the total surface area).

Neurotransmitter release was studied also for viable neurons after scratch and compared with standard cell cultures performed before on the same material, $[26,28]$ to study any possible change in function due to assays. The main feature of the neurons is the release of neurotransmitters to the synapse and the eventual activation of receptors that will trigger a physiological response. After the arrival of an action potential at the synapse, neurons undergo depolarization resulting in the release of neurotransmitters. In vitro, neuronal depolarization can be achieved by increasing the extracellular $\mathrm{K}^{+}$concentration in the media and subsequently the released neurotransmitter content is analyzed by HPLC. Immediately after scratch cell cultures were exposed $2 \mathrm{~min}$ to HBSS buffer containing $5 \mathrm{mM} \mathrm{K}^{+}$(basal condition) and subsequently to $90 \mathrm{mM} \mathrm{K}^{+}$(depolarization condition) to promote neurotransmitter release. The concentration of the main excitatory and inhibitory neurotransmitters (glutamate and GABA, respectively) was determined as described in [26]. In electric field stimulation experiments, neurotransmitter concentration was measured directly in HBSS buffer containing $5 \mathrm{mM} \mathrm{K}^{+}$, immediately after EF exposure, (no need of $90 \mathrm{mM}$ ).

Scratch assay: After 5 DIV, cells were mechanically transected using a plastic micropipette tip, generating a reproducible rectangular cell-free area $1 \times 22 \mathrm{~mm}$ long. [45] Filling of this empty space was measured evaluating the extent of new neurite growth by determining the corresponding tau fluorescent surface coverage of the scratch, in the absence and the presence of EF (performed immediately after the scratch). Spontaneous neurite outgrowth at the scratch zone was followed in time in culture conditions up to seven days after the lesion, in order to evaluate the optimal time for comparison of stimulated and non-stimulated cell cultures.

Using various anode/cathode configurations, EF effects on the re-filling of the cell-free area by neurites was measured, using the same delivered charge, on several scratched independent cell cultures placed near the anode of the cathode. Each case signifies a material, and electrode combination and an EF protocol. A parallel non stimulated system simulates all stages of the procedure and will be used as control in each experiment.

Measurements of the regeneration are made based on fluorescence from tau immunostaining of newly generated neurites, as described above. Data analysis is given as mean \pm sem of, at least, three experiments using independent cell cultures in each case, with an independent blank, which add to a total of 100 samples from 7 to 10 independent experiment 
types. First, electrostimulation experiments with charges above and below CSC are performed to determine the effect of surpassing the values of CSC for the coating material and the influence of the capacitive Pt part, and therefore to find the optimal $Q$ levels of the study. After that, EF protocols with charge control are based on potential control, or current control, with net charge dynamic pulses in the scale of seconds, with global $Q$ of $80 \%$ of the material with smaller CSC (bilayer). DC mode represents a charge delivery with pulses always cathodic with respect to the working electrode controlled by the Reference electrode (and anodic in the counterelectrode), while AC net charge experiments alternate pulses in both directions, with the cathodic being first and always with larger charge. Both DC and asymmetric AC (shown in Figure 5) represent net cathodic charges for the working electrode and anodic for the counterelectrode.

\section{Results and Discussion}

\section{Materials}

Table 1 summarizes the materials group on which a previous selection was made based on cell survival in neural cell cultures, $[18,26-28,30,46]$ as well as their electrochemical characterization ( $\mathrm{CV}$ and $\mathrm{Z}$ measurements) in the form of coatings. As reference, Platinum, IrOx and PEDOT-PSS are also shown. An additional list of tested material coatings among which some have been selected depending on neuronal survival is shown in Supplementary Material S1.

Thus, the materials chosen and used as coatings in this study reflect the possibility of intercalation of cations while sustaining neural adhesion and cell viability, and correspond to two types of structures, Polymers and Oxide/C materials: a conducting polymer bilayer and an IrOxgraphene hybrid: B-Lys, and IrOx-eG respectively. In particular, IrOx-eG is a truly nanostructured material, since its preparation is based on colloidal nanoparticles of IrOx attached to exfoliated graphene that are electrodeposited anodically in a similar procedure to that used for pure IrOx. The polymer bilayers on the other hand contain aminoacid lysine as counterion, and although the structure may also be considered nanostructured, the active part, the conducting polymer, is similar to other polymers. 
Table 1. Summary of Charge Storage capacities, CSC (Coulombs $\left./ \mathrm{cm}^{2}\right)$ and $Z(\Omega)$ for $1 \mathrm{~cm}^{2}$ electrodes 0.1 to $100 \mathrm{~Hz}$, for electrodes used and some additional materials shown for reference (CSC based on cycle 1 and after 1000 cycles, at 10 $\mathrm{mV} / \mathrm{s} ; 100 \mathrm{mC} / \mathrm{cm}^{2}$ are equivalent to $\approx 1000 \mathrm{~F} / \mathrm{g}$ for IrOx based materials. [18,26-28,30,46]

\begin{tabular}{|c|c|c|c|c|c|c|}
\hline Materials & $\begin{array}{c}\mathrm{CSC}_{\mathrm{c}} \\
\left(\mathrm{mC} / \mathrm{cm}^{2}\right)\end{array}$ & $\begin{array}{l}\mathrm{CSC}_{\mathrm{c}} \text { after } \\
1000 \mathrm{cycles} \\
\left(\mathrm{mC} / \mathrm{cm}^{2}\right)\end{array}$ & $\begin{array}{l}\text { Thickness } \\
\text { (nm) }\end{array}$ & $\begin{array}{l}\text { RMS } \\
\text { (nm) }\end{array}$ & $\begin{array}{c}\mathrm{Z}(\mathrm{OHz}) / \mathrm{Z}(100 \mathrm{~Hz}) \\
(\Omega / \Omega)\end{array}$ & Acronym \\
\hline Pt (therm. evap.) & 0.1 & 0.1 & - & 0.8 & $31600 / 125$ & Pt \\
\hline IrOx (I constant) & 10 & [6] & 100 & 10 & [49] & - \\
\hline $\begin{array}{l}\text { IrOx (dynamic } \\
\text { electrodep.) }\end{array}$ & 22 & 10 & 130 & 2 & $177 / 63$ & IrOx \\
\hline $\begin{array}{l}\text { PEDOT-PPy-Lysine } \\
\text { Bilayers }\end{array}$ & 3 & 4 & 160 & 16 & $891 / 100$ & B-Lys \\
\hline PEDOT-PSS & 6 & 4 & 350 & 20 & $398 / 63$ & PEDOT-PSS \\
\hline $\begin{array}{l}\text { IrOx-exfoliated } \\
\text { Graphene }\end{array}$ & 94 & 64 & 700 & 160 & $100 / 63$ & IrOx-eG \\
\hline
\end{tabular}

Cyclic voltammetries of the coatings obtained in this work are consistent with previous data. $[18,26-28,30,46]$ Within the water safe potential window at this neutral pH , (phosphate saline buffer described in the experimental part), in all cases, one or two redox processes related to intercalation in the coating material, IrOx, or conducting polymer based, are observed. We have proven before to correspond to cation intercalation during cathodic processes. For IrOx-eG, $\mathrm{Na}^{+}$(hydrated) intercalation occurs in the cathodic sweep simultaneous with $\mathrm{Ir}^{+4}$ reduction, and $\mathrm{Na}^{+}$desintercalation and $\mathrm{Ir}^{+3}$ oxidation in the anodic reversed sweep. [18] $\mathrm{Q} / \mathrm{m}$ ratios from quartz microbalance studies have shown the abundant $\mathrm{Na}^{+}$ion intercalates as a hydrated species during reduction, although $\mathrm{H}^{+}$can never be discarded. [18]. Polypyrrole and PEDOT redox changes occur within the carbon conducting chains during cathodic and anodic sweeps also with simultaneous intercalation of hydrated $\mathrm{Na}^{+}$ions, while lysine is never released. Scheme 1 summarizes the processes of both materials.

The coatings also yield the expected CSC values, $[18,26-28,30,46]$ for the specific areas and thicknesses prepared here. During use (redox sweeps up to 1000 cycles) the electrode capacity remains at $\approx 70 \%$ of the original in the materials chosen, which is also one of the reasons for the selection of certain materials. Significantly, materials containing conducting polymers never surpass the value of $20 \mathrm{mC} / \mathrm{cm}^{2}$. However we have chosen one of them, bilayer PEDOT-polypyrrole-Lysine aminoacid, because is the polymer with complete reproducible behavior in previous long term neural cell cultures. [30] Not surprisingly, the better conductors also result in thicker deposits and larger capacities since all volume is accessible during 
intercalation redox processes (Figure 1). As observed, CSC is much larger and impedance lower for IrOx-graphene hybrid. While IrOx electrodeposition is self-limited, when graphene is present in IrOx-graphene nanoparticles, the conductivity of the resulting coating allows thicker deposits.

IrOx-eG

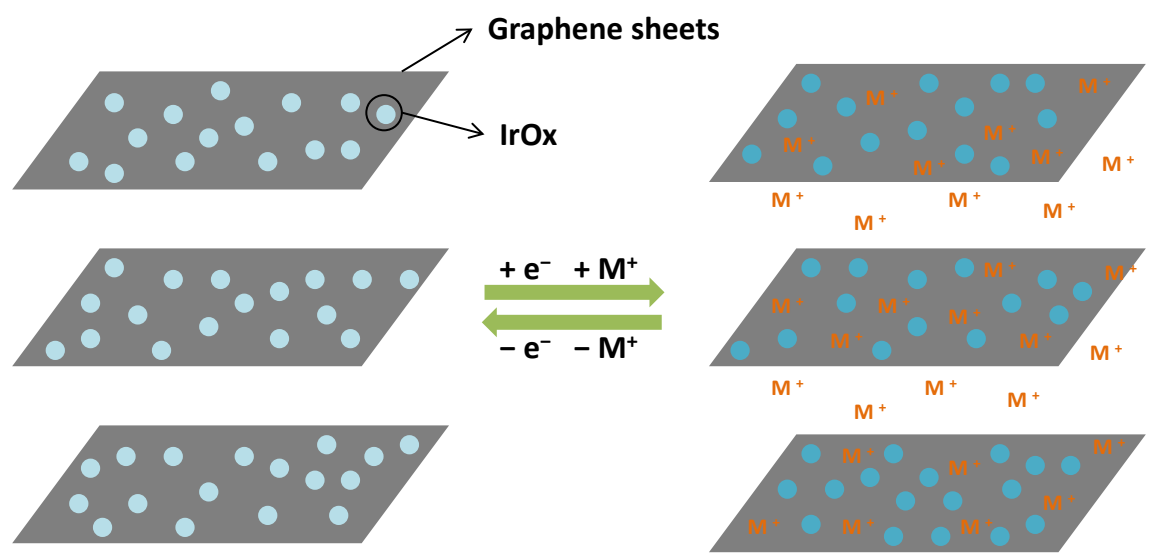

Polymer bilayer
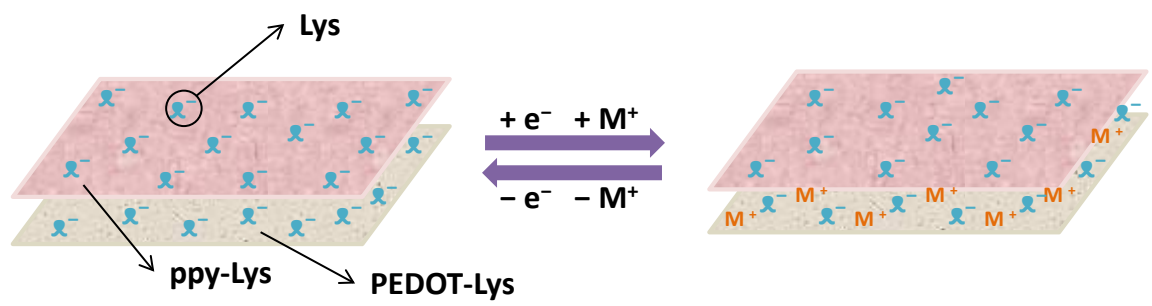

$\mathrm{M}=\mathrm{Na}, \mathrm{K}, \mathrm{Ca} \ldots$ etc

Scheme 1. Intercalation processes for Nanostructured IrOx-eG and bilayer polymers. Cathodic behavior corresponds to the reduction of iridium and intercalation of $\mathrm{Na}^{+}$while anodic processes would correspond to oxidation and deintercalation of cations. Also possible and competitive are intercalation of other cations o deintercalation of anions such as $\mathrm{OH}^{-}$from the oxide during cathodic processes. Anodic processes are the reverse reaction. Iridium starts from +3.5 valence and has both paths available, while the polymer must be pre-reduced to have both paths at the start of experiment.

It is worth noting that although CSC values may increase when roughness increases, as reported for platinum black, [50] the magnitude of the increase in IrOx-graphene with respect to IrOx is significantly larger than the expected from roughness variations observed in Figure $1 \mathrm{C}$. Beyond the carbon usual capacitance, nanostructuring creates a multiplying effect with capacities one order of magnitude larger, possibly because IrOx redox processes are more accessible during EF stimulation than in pure IrOx. Because we are dealing with nanostructured intercalation materials, where the whole coating volume is involved in the charge transfer process, it is of interest to describe the value of charge capacity in $\mathrm{mC} / \mathrm{cm}^{2}$ also as specific capacitance (F/g). As compared with PEDOT-PSS-graphene hybrids published before with 100 to 
$190 \mathrm{~F} / \mathrm{g}$, [51] the $\approx 100 \mathrm{mC} / \mathrm{cm}^{2}$ value for IrOx-graphene hybrid with $700 \mathrm{~nm}$ thickness corresponds to $\approx 1020 \mathrm{~F} / \mathrm{g}$ while reduced graphene oxide has been reported to have $82 \mathrm{~F} / \mathrm{g}$ capacitance, and IrOx $100 \mathrm{~F} / \mathrm{g}$. [51]

A
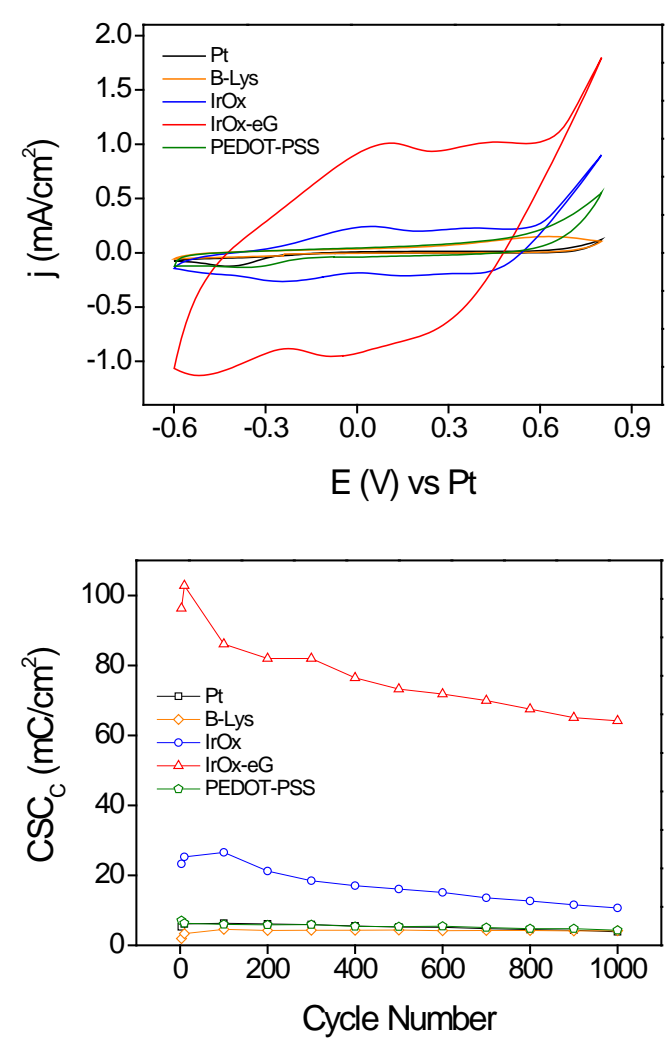

C
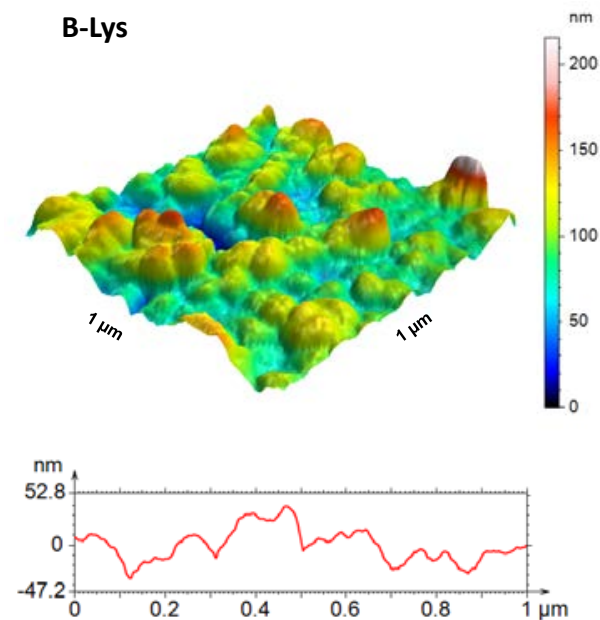

B

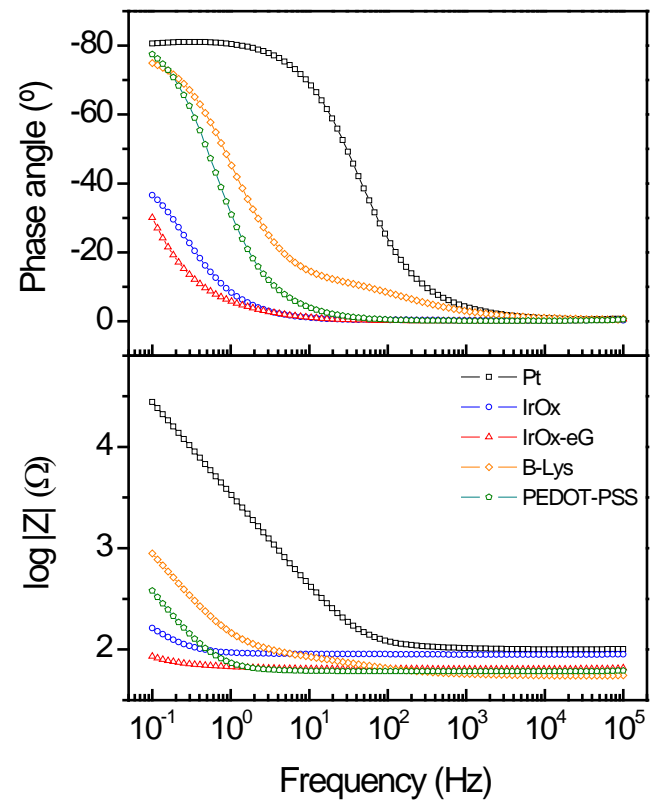

IrOx-eG
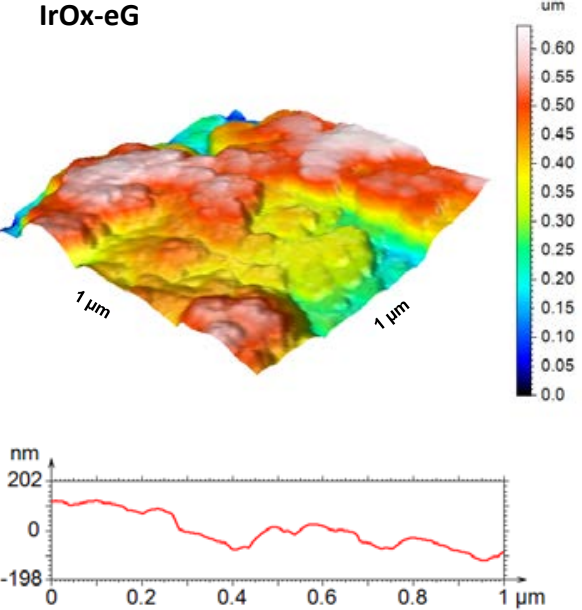

Figure 1. Comparison of A) Cyclic voltammetry (top) and Charge capacity stability (bottom) and B) Impedance and Phase vs frequency for each of the electrodes selected in EF assays: Pt, Bilayer PEDOT-polypyrrole-Lysine (B-Lys) and IrOx-exfoliated Graphene (IrOx-eG). C) AFM Microstructure comparison of B-Lys and IrOx-eG coatings on Pt. Below: representative profiles for each coating. Note that Pt surface has a roughness below the $\mathrm{nm}$ scale (not shown). 
Although the CSC comparison among materials is necessary to evaluate the electrochemical response and the possible EF effects on neurite growth on scratched cultures, for a true comparison the experiments are based on the same charge injected during the EF protocol and therefore help differentiate the intrinsic effect of each electrode material.

A significant aspect in bioelectrodes is the actual impedance of the electrode and interface global system. $[29,50,52]$ Impedance for each of the coated electrodes is measured in a wide range of frequencies and shown in Figure 1B, as compared with $\mathrm{Pt}$ and other single phases as IrOx and PEDOT-PSS. Figure 1B evidences significant differences in $|Z|$ among coatings, both at low frequencies and up to $10^{2}-10^{3} \mathrm{~Hz}$. In all cases, the coated platinum electrodes show lower impedance than the bare platinum, with differences of several orders of magnitude, $\left(10^{3}-\right.$ $10^{2}$ vs $3 \cdot 10^{4} \Omega$ respectively) at low frequencies. At $0.1 \mathrm{~Hz}$ the lowest impedance materials are IrOx and IrOx-graphene hybrids. At $1 \mathrm{~Hz}$, PEDOT-PSS and IrOx-eG gets equal in impedance, and at 100 $\mathrm{Hz}$, B-Lys reaches the same value than IrOx-eG and PEDOT-PSS. Above that frequency, conducting polymers and IrOx-eG continue being the lower impedance systems while IrOx has larger impedance (100 $\Omega$ for IrOx to $63 \Omega$ for polymers and IrOx-eG).

Phase angle data is directly related with the impedance results discussed above. All coatings have lower phase angle than Pt and two groups of coatings can be found. At low frequency the phase angle for conducting polymers is similar to that of bare platinum underneath the coating. Polymers undergo a transition to capacitive behavior at $1 \mathrm{~Hz}$, while Pt shows it the $10^{1}-10^{2} \mathrm{~Hz}$ regime. On the other hand, the lowest impedance materials at low frequency, IrOx and IrOx-eG, behave in all frequency range basically as pseudocapacitor, as expected from their intercalation redox reactions. Although these reactions also occur in conducting polymers, they don't show the same contribution in impedance measurements, evidencing a different intercalation mechanism among polymers and IrOx based materials.

The relative ordering of impedances occurs up to frequencies slightly above the classical ones used in functional stimulations like Parkinson or neural recordings $[5,20,29,53,54](100 \mathrm{~Hz}-$ $1 \mathrm{KHz}$ ) and so, the results shown in this paper maybe of interest in these fields also.

The low impedance for chosen materials represents a property of the material as electrode but also a significant lower resistance in the charge transfer at the interface. This may be so for various reasons. The simplest cause may arise because the effective surface is increased due to roughness (Figure 1C), in a similar way as described above for CSC. Table 1 shows that BLys and IrOx-eG have larger roughness than Pt or IrOx. Using platinum black, Z values are greatly decreased $v$ s bulk Pt but the decrease in impedance is not of the magnitude found for IrOx and PEDOT-PSS coatings. $[50,55]$ Therefore other factors apart from roughness exist, the main being the fact that an alternative charge transfer mechanism occurs at the interface, the intercalation process, not available for platinum, and that the redox sites are largely available in the nanostructured hybrid material. In terms of the comparison for various frequencies, the different impedance order is probably related to the different kinetics of intercalation processes in the 
various types of materials, being very different in IrOx or conducting polymers, basically because of different structural changes during intercalation.

This materials characterization adds to the described selection of certain materials to study the effect of EF on primary cultures of cortical neurons. Pt as an usual commercial electrode, IrOx-eG as the material that best perform in terms of CSC and impedance and the bilayers PEDOT-polypyrrole-Lysine aminoacid, both with fully reproducible behavior in the survival of neural cell cultures. [28,30]

\section{EF Assays related to Charge delivered}

In order to optimize the electric field protocols useful for a regeneration in vitro model, the work has defined the optimal charge to be delivered. An initial electric field assay is performed for charge deliveries above and below CSC values on a normal cell culture with no scratch done.

A

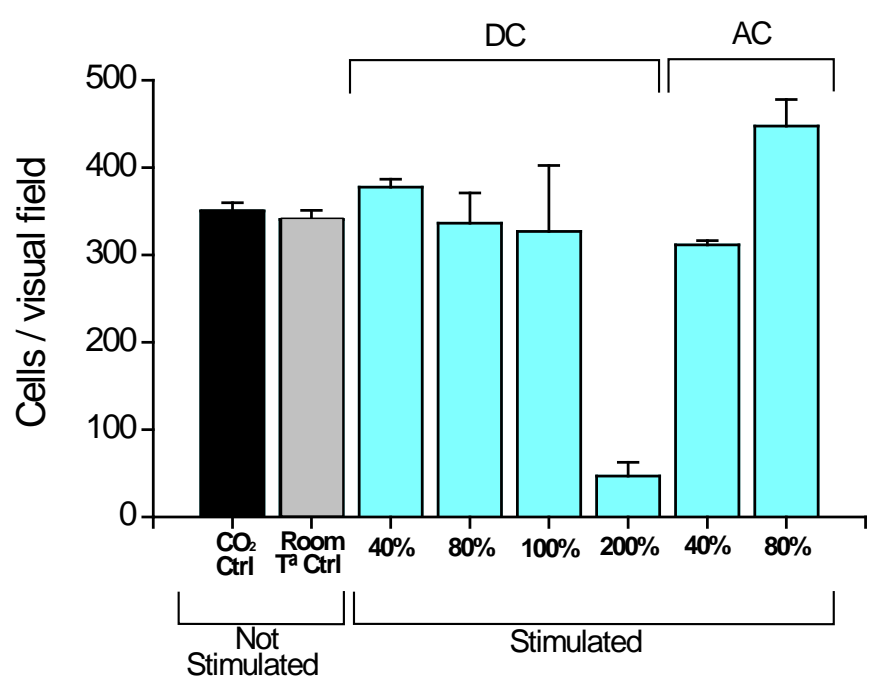

B
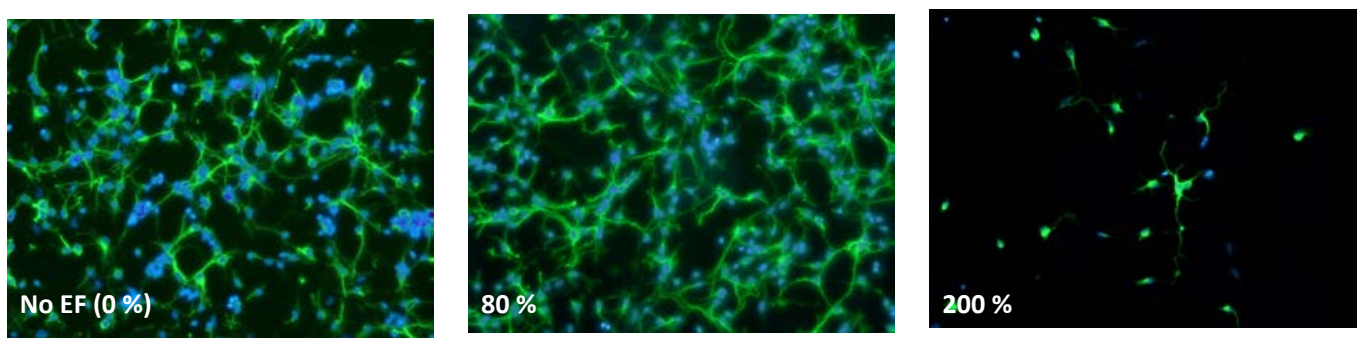

Figure 2. A) Neuronal survival after Q/CSC delivery in the close vicinity of culture dish using a B-Lys electrode. \% values represent the relative charge used vs CSC. All assays below $100 \%$ are viable. B) Fluorescence microphotograph showing Tau immunostaining (green) and bis-benzimide staining (blue) of cortical neuron cultures near B-Lys electrodes in absence or presence of EF: no EF (Q O \%) , $80 \%$ and $200 \%$ of CSC. Applied EF protocols are described below. 
As observed in Figure 2 for B-Lys electrodes, neural cells near the electrode are viable either in absence or in presence of EF, as long as the charge injected in the experiment is maintained below the faradaic charge capacity limit, CSC, while the viability declines significantly when the charge goes beyond that value. These experiments are a proof of the proposed concept, when the electrode behaves as intercalation electrode, the charge injected does not damage the cells, while for charge values above that capacity, only the capacitor mode acts and other secondary reactions may occur. Therefore, the scratch-EF assays are made with charge control so that $\mathrm{Q}<\mathrm{CSC}$ in all of them. It is important to mention also that no differences are observed among the various tests, either inside $\left(\mathrm{CO}_{2} \mathrm{Ctrl}\right)$ and outside (Room Temperature Control) the incubator chamber within the time span of experiments (40-60 min max.). The position of the salt bridge is optimized to assure homogeneous cell cultures, and no differences are observed among different zones of the culture.

\section{Spontaneous lesion repair in vitro}

In order to establish the influence of electric field stimulation on neurite outgrowth, spontaneous regeneration of neurites in the cell free area needs to be established in absence of field. For that, regeneration is measured in absence of field, immediately ( $\mathrm{T}=0 \mathrm{~h}$ ) and during a daily time course following the scratch, that shows the optimal time for comparisons with EF studies. Figure 3 shows the time course of regeneration of the scratched zone after scratching the neuronal monolayer at 5 DIV, with clear differentiation of neurites and nuclei (Figure 3B). Note that the neuron viability is not altered in the zone far from the lesion, and that the neurons nuclei do not migrate through the lesion zone, while neurites extend towards the opposite side. After 7 days the emitted neurites cover the whole area and seem to connect (Figure 3A). As observed in Figure $3 \mathrm{C}$, the initial recovery of the zone is larger during the first days, and it stabilizes around day 3. Eventually, neurites grow until they contact neurons growing from the opposite side of the scratch around day 7 after the scratch. In all cases, cell nuclei do not seem to migrate through the scratch, and only neurites extend. Fitting the results to a sigmoid curve, $50 \%$ of recovery was obtained at $2.4 \pm 0.4$ days $(n=4)$ and, therefore, day 2 after the scratch is chosen as the best to study the electric field effects using various electrodes. Furthermore, neuronal viability was not impaired in the non-wounded area and comparable neurotransmitter release was observed to cases with no scratch (Figure 3D). An increase of tau immunoreactivity in scratch edges is observed evidencing a scar-like line formed. 
A

Time After Scratch
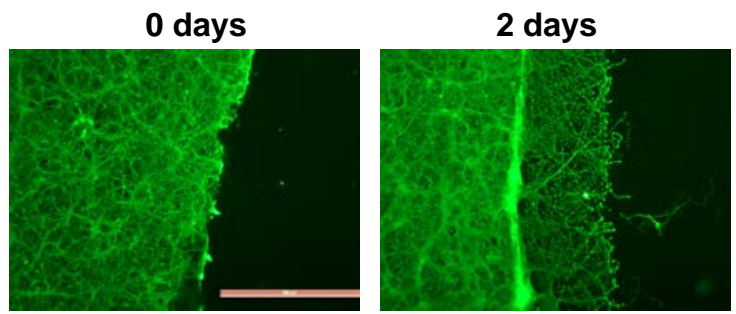

4 days

7 days
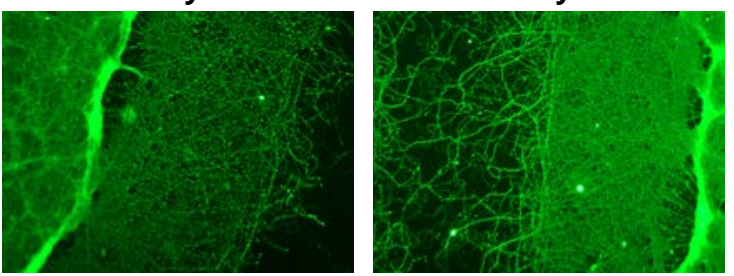

B
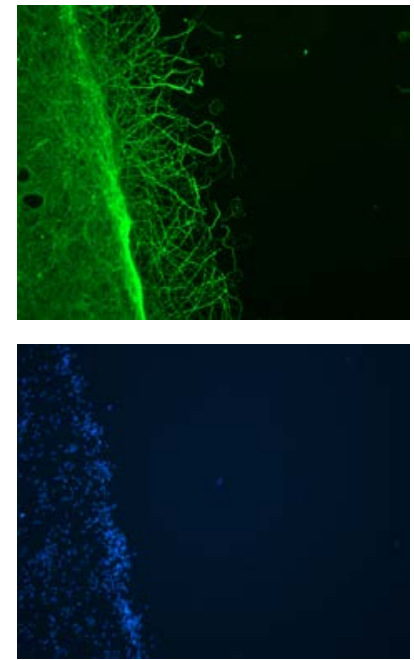

C

Time Course Scratch

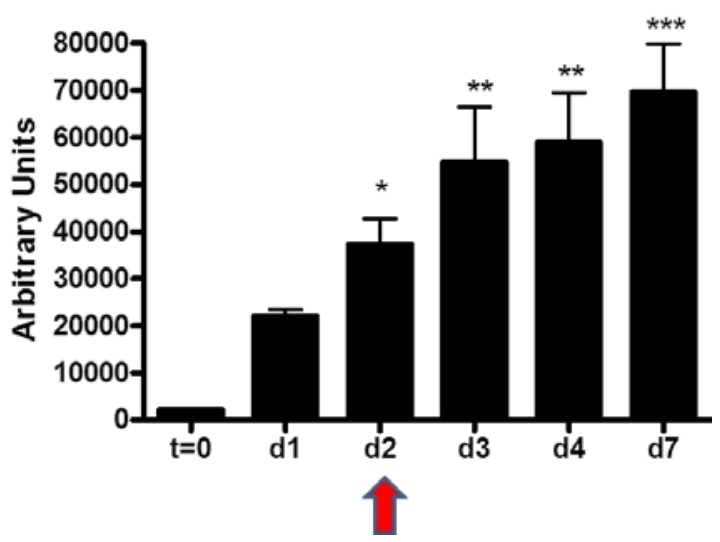

GABA release

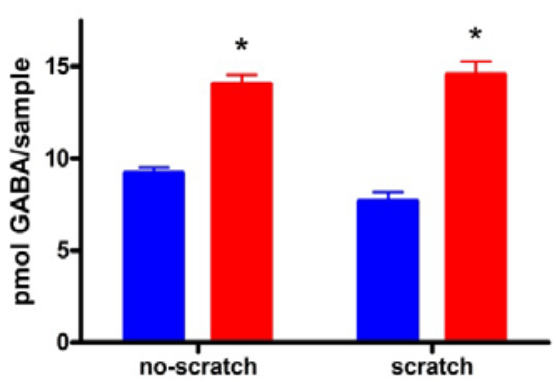

Figure 3. A) Tau imunostaining of scratched cortical neuron cultures showing spontaneous regeneration of surface covered by new neurites after scratching the neuronal monolayer at 5 DIV. B) Neurite and nuclei images for comparison (Cells were processed for Tau inmunocitochemistry and Bis-benzimide staining of the nuclei). C) Quantitative integration of the area covered by new neurites at different days after scratch. Results are mean \pm sem ( $n$ $=5) .{ }^{*} p<0.05{ }^{* *} p<0.01$ vs $t=0$ after significant one-way ANOVA. D) Glutamate and GABA release after the scratch lesion was performed. Cultured cells were exposed to a basal HBSS solution (containing $0.005 \mathrm{M} \mathrm{K}^{+}$; blue bars) or to a depolarizing HBSS solution (containing $0.09 M K^{+}$; red bars). Results are mean $\pm s d, n=3$. ${ }^{*} p<0.001$ vs $0.005 \mathrm{M} K^{+}$-HBSS solution after 2-way ANOVA followed by Bonferroni's posttest. 


\section{Electric field stimulation on scratched cell cultures.}

Electric field application is performed with the setup shown in Figure 4, where one borosilicateH coverslip with a scratched cell culture is placed near the anode and another one near the cathode.

Table 2. Cathode/anode combinations in EF experiments for scratch cell cultures.

\begin{tabular}{cccccccc}
\hline $\begin{array}{c}\text { Cathode } \\
-\end{array}$ & Pt & Pt & B-Lys & B-Lys & B-Lys & IrOx-eG & IrOx-eG \\
\hline $\begin{array}{c}\text { Anode } \\
+\end{array}$ & Pt & $\begin{array}{c}\text { B-Lys } \\
\text { reduced }\end{array}$ & Pt & B-Lys & $\begin{array}{c}\text { B-Lys } \\
\text { reduced }\end{array}$ & Pt & IrOx-eG \\
\hline
\end{tabular}

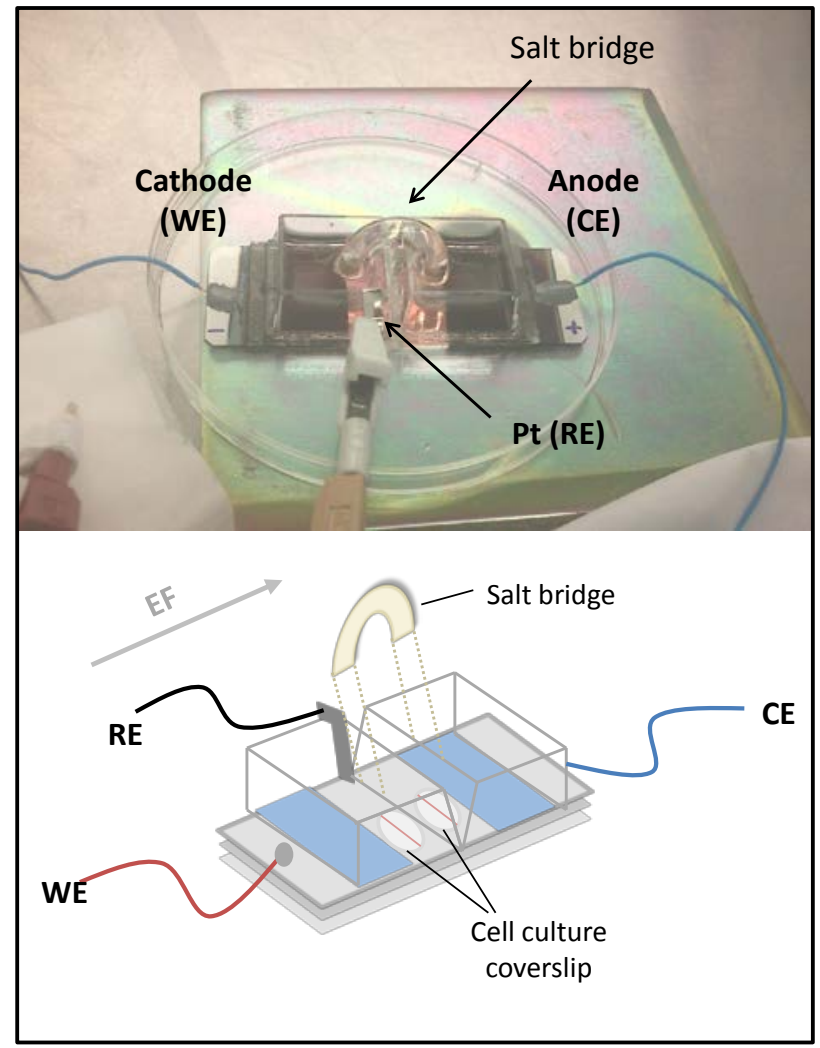

Figure 4. Image and schematic representation of the device (Electrochemical cell) used with electrodes coated with various materials. Cell cultures on borosilicateH coverslip are placed near each electrode once scratched, for EF application. Each culture plate is returned to its original well-plate in the incubator after the process. The combinations of cathode (-) and anode (+) are shown in Table 2.

As shown in Figure 4, the various materials are deposited on Pt coated glass on both ends of the glass piece, leaving an uncovered center part where the plastic barrier creates two zones, 
one near the cathode and the other near the anode. It is in each of those two zones where the cell culture is placed after scratch, for EF experiments. A parallel non stimulated system is reproduced in exact same conditions and simultaneously and will be used as control in each experiment. Both sides of the electrochemical cell are connected by a U-shaped glass salt bridge filled with the same electrolyte (Hank's buffer solution) and agar-agar gel, to prevent migration of possible byproducts from one half cells to the other, in an optimized position to prevent inhomogeneities of the field. A plastic holder used for cultures is placed on top of the glass and is sealed at the external part with fish tank silicone to prevent liquid to leak out and outer contamination to enter the culture zone.

As shown in Table 2, the coatings are based on bilayer PEDOT-polypyrrole Lysine as prepared in both cathode and anode or reduced previously if used at the anode, and on IrOxgraphene in both anode and cathode. A Pt pseudo-reference electrode controls the potential of one of the electrodes during constant potential experiments. It is worth mentioning, for comparison with previous work, that the present set up creates a global electrochemical cell potential of 50 to $100 \mathrm{mV} / \mathrm{cm}$, as in previously Xenopus reported experiments with external electrodes. $[1,2,10,11,56]$ Also, blanks of EF applied with no scratch evidence that the electric field effects are the same in all sections of the cell culture.

Once scratched, the cell culture coverslips are placed in the anode and the cathode side taking special attention that the cell free area is oriented perpendicularly to the electric field direction to evaluate the effect in the direction of neurite growth.

Pulsed electric fields are applied in DC and asymmetric AC-net charge modes, by controlling potential or current, and limiting the final charge delivery to a value below the CSC of the bilayers (see Figure 5). Specifically, charges $80 \%$ of the bilayers CSC value has been chosen to minimize possible heterogeneities of the material or errors in CSC. To facilitate comparison among materials, experiments with IrOx-graphene are performed with the same charge delivered than those with B-Lys, even though they could be expanded longer in time. That charge corresponds to $10 \%$ of the CSC for the IrOx-eG material.

Pulses are intended to relieve stress and allow heat dissipation, but maintain the DC character of the experiment, since the final purpose aims for possible repair or directioning effects. The interval of pulses is therefore in the seconds range with frequencies in the $0.01 \mathrm{~Hz}$ regime. In the case of $A C$, the reverse pulse is defined to be about $10 \%$ of the forward pulse. Thus, for B-Lys coatings, a net charge $80 \%$ of $20 \mathrm{mC} / \mathrm{cm}^{2}$ is applied in about 40 minutes. In this configuration, intercalation processes in the coating material are in the appropriate range for the redox process, and therefore the capacitive part of the electrode is minimized. Clearly, the comparison with Pt electrodes represents a comparison with a case of no intercalation, with radical formation and much lower CSC. 


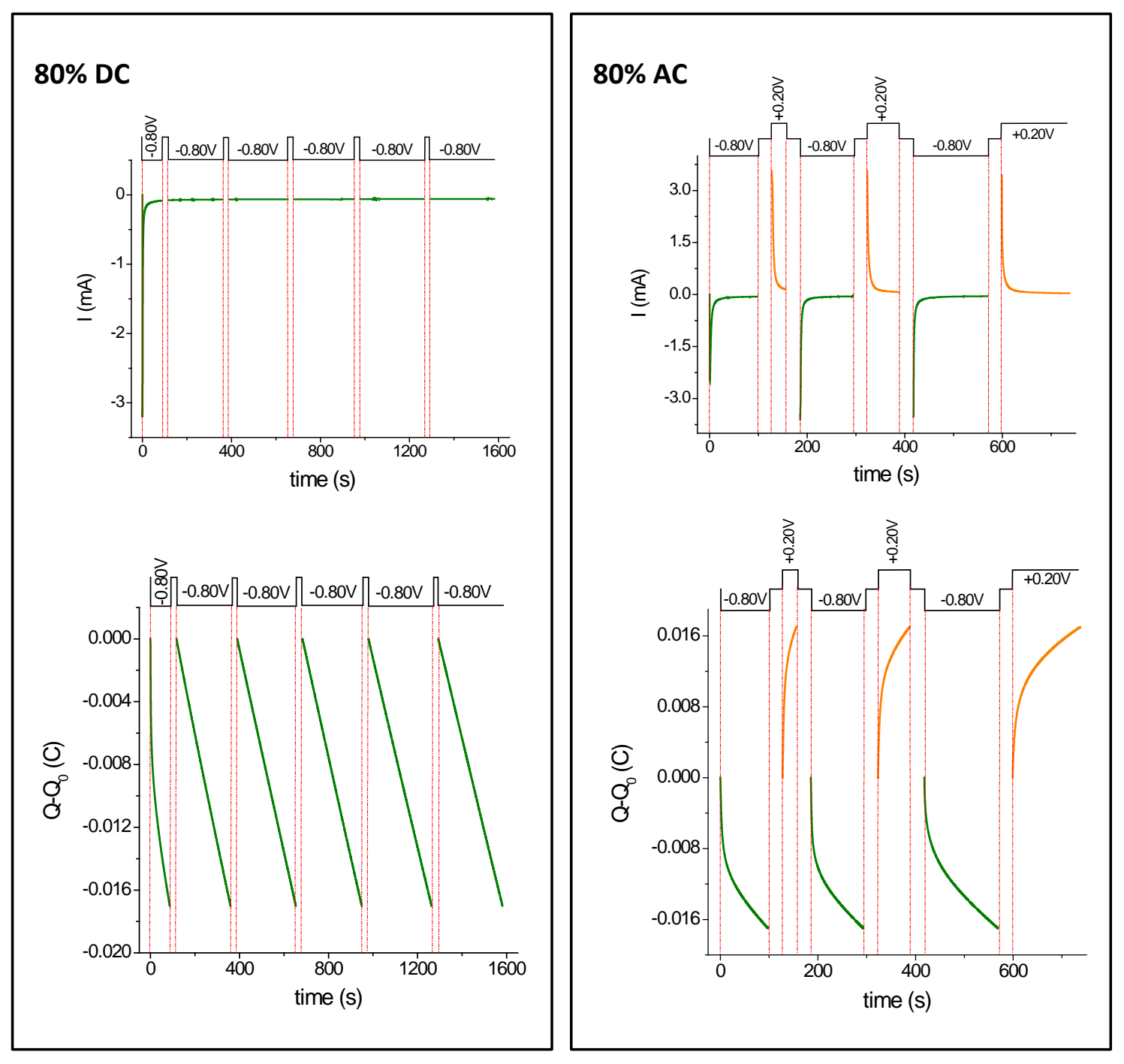

Figure 5. EF protocol examples in DC (80\% CSC) and AC ( $80 \% \mathrm{CSC})$ modes at constant potential ( $V$ vs Pt) and their current (I ( $m A)$ ) response along with $Q$ (Coulombs) delivered. Pulse is $Q$ limited in pre-established fractions of $80 \%$ CSC. Delay times have been chosen to be $30 \mathrm{~s}$. Net cathodic charges refer to the electrode being controlled in the threeelectrode cell (cathode), and corresponds to the same charge at the anode.

After EF stimulation, cell culture coverslips are returned to the optimal culturing conditions in their own culture medium and are allowed to evolve 2 days, before analyzing their neurite extension in the scratch zone in both sides, anode and cathode. In order to detect the extension of repair in the lesion zone, cells were fixed with paraformaldehide, and inmunocitochemistry markers for the cytoskeleton (Tau protein) images were analyzed with Image program.

The results show non-significant differences between DC and AC-net charge experiments, as expected for the actual charge delivered is the same. Statistics however are taken from equal DC experiments to prevent undetected parameters. In all selected cases, there is in vitro regeneration at the scratch zone, both in absence and in presence of electric field. In all, neurites extend in an organized manner and there is an increment of immune reactivity against tau in 
what resembles a scar zone. Beyond that zone, no nuclei migrate, and all remain adhered in nonscratched area of the culture. So, neurite extension towards the opposite side of the scratch suggests the existence of chemical signals that are being detected. Both sides of the scratch grow in a non distinguishable manner, and in both scratches, near the anode and near the cathode (See Figure 6).

A

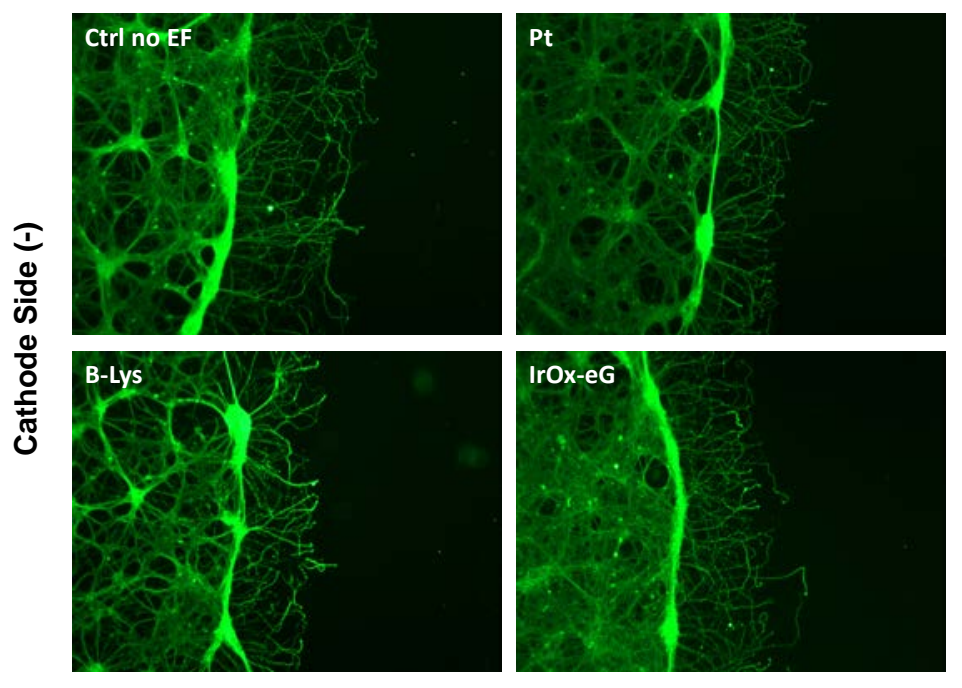

B

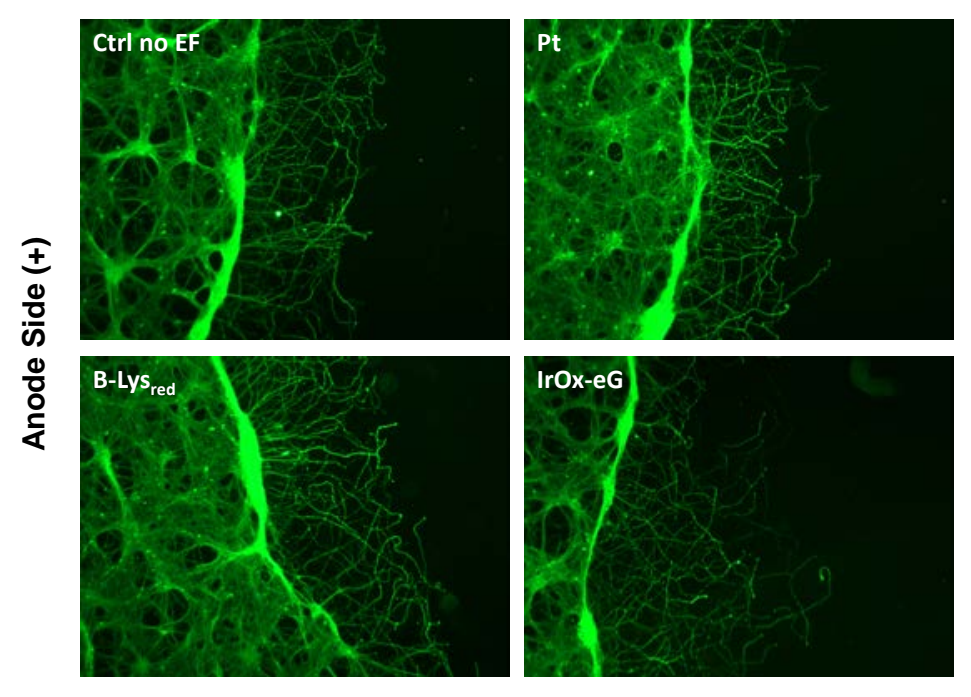

Figure 6. Fluorescence microphotograph of Tau imunostaining in scratched cortical neuron cultures showing neurite extended spontaneously and in presence of EF, using various electrodes: uncoated Pt, bilayer PEDOT-polypyrroleLysine coating platinum, with Q delivered $80 \%$ of the CSC values, and IrOx-Graphene coating Pt electrode with equal $Q$ delivered: A) Cathode side and B) Anode side.

Figure 6 allows an easy visualization that there are differences in neurite extension over the scratch zone, with the same charge delivered, depending on the electrode material used, the 
stimulation with Pt electrodes being the less effective even in comparison with the spontaneous neurite outgrowth in the absence of electrical field (Control). That is so, even considering the small period of stimulation (about 25 minutes).

The summary of all experiments statistically averaged are shown in Figure 7A, for both the anode side and the cathode side in each electrode combination. Two-way analysis of variance reveals an overall prominent effect of the type of material on the newly regenerated neurite area at the scratch zone $(p<0.001 ; F=6.26 ; d f=4)$ and a marginal difference for the anode/cathode zone $(p=0.07 ; F=3.66 ; d f=1.29)$. Neurotransmitter release show no differences among standard cultures published before and this scratch model and are not shown here, implying that the model does not decrease neuronal function.

Noticeably, the recovered area was significantly increased in the anode (zone + ) when the stimulating electrodes were IrOx-eG ( $p<0.05$, one-tailed Student's t-test), $115 \%$ with respect to the spontaneous recovery in the absence of electric field. On the contrary, stimulation with Pt electrodes resulted in considerable decrease of the neurite extension in both the anode $(\approx 80 \%)$ and the cathode $(\approx 65 \%)$ ( $p<0.05$ and $p<0.01$, respectively, two-tailed Student's t-test).

Although not statistically significant, bilayer conducting polymers, B-Lys, result different if the electrode to be used as anode is previously reduced yielding a double span of available charge delivery at the anode and a double value of capacity for the global electrochemical cell. An improvement is observed for a pre-reduced anode B-Lys with respect to the use of both cathode and anode B-Lys as prepared in the oxidized form, showing neurite outgrowth $>95 \%$ in both anode and cathode with respect to the spontaneous case.

Also, neurite growth with IrOx-eG results better than with B-Lys electrodes and the difference is statistically significant in the anode ( $p<0.05$, one-tailed Student's t-test). Larger deviations are observed in the polymer cases with respect to IrOx-graphene (mean coefficient of variability $15.4 \%$ and $8.2 \%$ respectively), which gives relevance to the in vitro model proposed. Thus, the use of IrOx-eG results in neurite outgrowth covering near two times more surface than if Pt electrodes are used, for the same charge delivered and very small stimulation times.

Not surprisingly, the material with largest CSC values and lowest impedance by far in this frequency range, is the one with optimal results found during EF stimulation. It is also the case study in which CSC has been used only in $10 \%$ of the possible limits.

Although not shown, if anode and cathode have different coating materials, the results are more erratic, but are consistent with the fact that the lowest capacity electrode limits the counterelectrode. Thus, when Pt is in one of the electrodes (no coating), we also observe worst results globally, and always more in the cathode.

On the other hand, Figure 7 shows, for cathode and anode based on the same material, a trend for a slightly larger neurite extension in the anode side $(+)$ vs the cathode side $(-)(p=0.08$, two-tailed paired Student's t-test). This fact points to a small secondary reaction present only in 
the cathode side, and therefore $\mathrm{O}_{2}$ reduction is suspected to be the cause of some degree of radical formation. Usual indicators for radical detection are not compatible with the potential that results during EF application and no further demonstration has been attempted here in this sense. However, an additional indication comes from the fact that Pt electrodes show small cathodic waves in this media at $-0.4 \mathrm{~V}$ vs Pt (see Figure $1 \mathrm{~A}$ ), that disappear in presence of $\mathrm{Ar}$, giving support to the existence of $\mathrm{O}_{2}$ reduction at the cathode when no intercalation material is available.

An additional explanation may come from the ion depletion resulting at the interface derived from the intercalation processes that the materials undergo. $\mathrm{Na}^{+}$intercalation and desintercalation has been proven in IrOx, [18] and $\mathrm{H}^{+}$and $\mathrm{OH}^{-}$processes are known to be possible. As the cathode intercalates $\mathrm{Na}^{+}$, the anode may be desintercalating $\mathrm{H}^{+}$, in equivalent amount since they undergo reactions with the same charge. The variation of concentration for the small charge delivered may be calculated from coulombs law and it is very small (in the order of $1 \%$ of the total concentration). Intercalation-related depletion of ions may be responsible of the differences observed between cathode and anode, but given the effects observed when $Q$ delivered is above CSC values, $\mathrm{O}_{2}$ reduction is a more plausible explanation for those differences.

It is worth to remark here that electrodeposited IrOx has been proven to be an iridium oxohydroxide with $\mathrm{K}^{+}$intercalated when prepared, and that upon soaking and sterilization, no $\mathrm{K}^{+}$ remains. Also, iridium is in a formal oxidation state of +3.5 and has room for both oxidation and reduction (to +4 and +3 respectively). [18] On the other hand the conducting polymers are prepared in the oxidized form and thus have no room for acting as anode unless they are previously reduced as shown above. [30] as in some of the experiments. In all cases, an ionic gradient exists because of the presence of the electric field, which may act as guidance cue for neurite extension. The small differences observed in both sides of a scratch and in the anode and cathode sides, however, suggest that this gradient is not being the main factor in neurite extension. Furthermore, since experiments are compared for the same charge delivered, the process at the cathode (or the anode) involve the same ionic gradients and changes for all intercalation materials. 
A

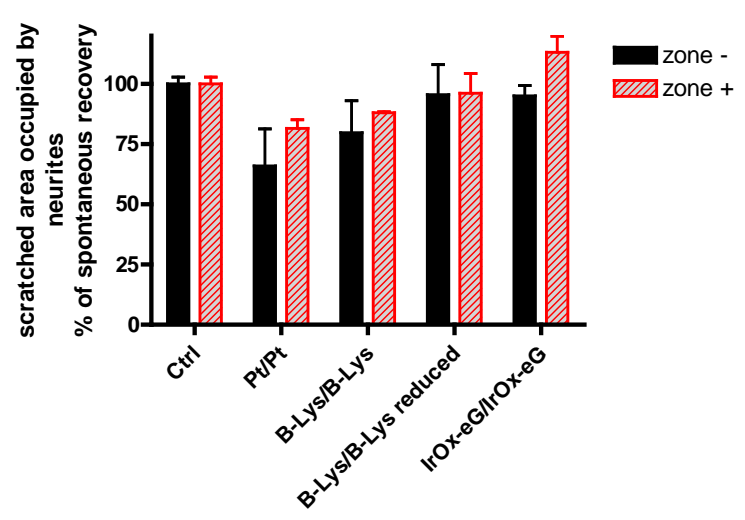

B

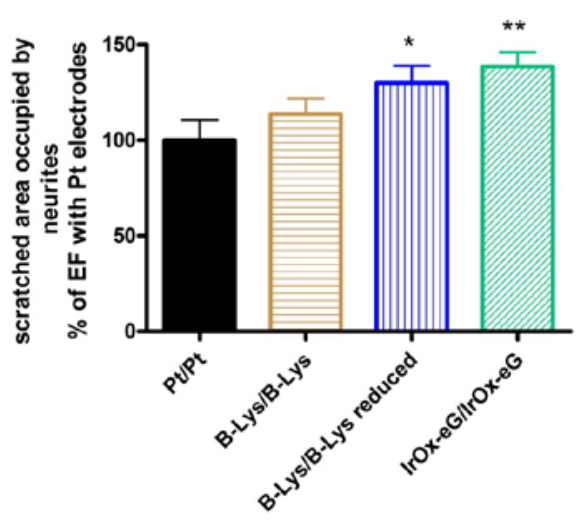

Figure 7. A) Quantification of new neurite growth at the scratch zone relative to the specific control with no EF for each experiment. In all cases shown, EF was DC with protocols defined in Figure 7 and charge delivered was $80 \%$ of the CSC for the bilayer PEDOT-polypyrrol-Lysine, which represents $10 \%$ of the CSC for IrOx-Graphene. Two-way Analysis of Variance. Global statistics: highly significant for electrode's material ( $p=0.0009)$ and non-significant for zone $(p=0.0658)$. B) $D C$-stimulated neurite regeneration in the scratch positive and negative areas with different electrode materials with respect to Pt/Pt electrode system. Results are mean \pm sem. ${ }^{*} p<0.05$ and ${ }^{* *} p<0.01$ after significant one-way ANOVA ( $\left.p<0.05 ; F_{3,15}=3.486\right)$ and post-comparison tests.

Despite the previous observation, no statistical significant difference were found between cathode and anode after both paired Student's t-test and two-way analysis of variance $(p>0.05)$. For that reason we have grouped neurite outgrowth data from both sides (Figure 7B) and compare all electrode's material with respect to the most common electrode in clinical practice (Pt electrode). Figure 7B shows a statistically significant increase of neurite regeneration when IrOx-eG/IrOx-eG ( $<<0.01)$ or B-Lys/B-Lys reduced $(p<0.05)$ combinations are used, clearly evidencing that neurite regeneration is improved with the material with larger CSC. Therefore, if $\mathrm{EF}$ is to be applied, the best is to coat the platinum electrode with an intercalation material, as the ones reported in this work. Voltammetries of the coatings after the stimulation/cell cultures evidence no significant lowering of capacities or currents, evidencing a very small degree of encapsulation within that time frame. 

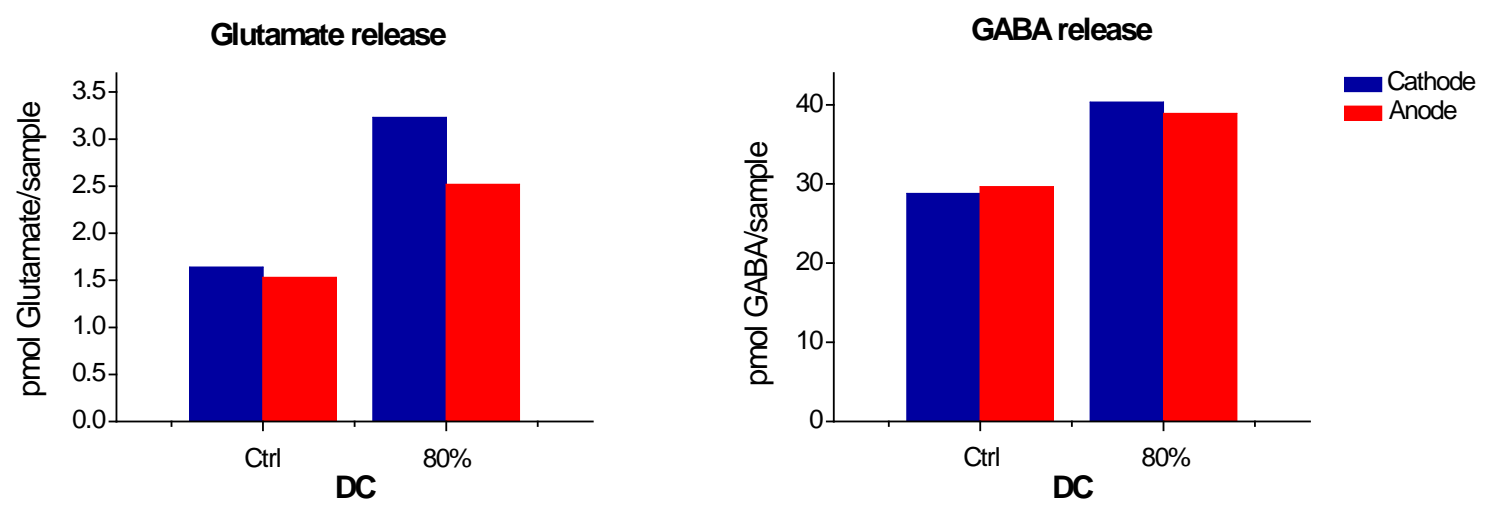

Figure 8. Quantification of Glutamate and GABA release in HBSS solution (containing $0.005 \mathrm{M} \mathrm{K}^{+}$) immediately after EF stimulation with Q $80 \%$ of the CSC values, for B-Lys electrodes, at both cathode and anode zones.

Neuron function is evaluated, in an initial study, for a set of samples representative of the EF stimulation, by GABA and Glutamate release for $80 \%$ CSC charge delivery. (See Figure 8). Although not covering all samples, the $80 \%$ CSC charge stimulation considered safe, indicates clearly that the electric field stimulates both neurotransmitters release above the reference found for usual cell cultures. An increase of $197 \%$ and $165 \%$ for glutamate was observed for glutamate, while $140 \%$ and $131 \%$ increase was found for GABA, at the cathode and anode respectively. Changes are, therefore observed in both sides of the electrochemical cell, and so, seem related to the ionic gradient created by the EF. Observations with Platinum electrodes are similar, and therefore neurotransmitter release, as opposed to neurite outgrowth, does not seem affected by the secondary reactions at the Pt interface, but rather by the existence of the field. Previous reports in deep brain stimulation have shown also release of GABA and Glutamate induced by the applied electric field, possibly through axon polarization and opening of $\mathrm{Ca}+2$ channels. [57] Thus, although scratch did not affect neuronal function, EF stimulation does increase neurotransmitter release, independently of electrodes used, as also observed in in vivo experiments.

McCaig reported neurite directioning towards the cathode (60\%) in individual cells from Xenopus laevis neurons $[1,2,11,58]$, and also in hippocampus mammalian dendrites but not axons, while others migrated towards the anode. The charge of the substrate has also been shown to modify the direction of galvanotropism for embryonic frog spinal neurons; neurites grew toward the negative electrode (cathode) on negatively charged tissue culture plastic or laminin, whereas neurites growing on positively charged poly-L-Lysine turned toward the positive electrode (anode). [5]

Beyond the single cell study, there have been limited studies of electrically stimulated mammalian neurons cultures, and these studies focus primarily on hippocampal neurons in the CNS in the absence of glia. In a graded response to constant DC fields of 28 to $219 \mathrm{mV} / \mathrm{mm}(24 \mathrm{~h}$ duration), embryonic rat hippocampal neurons responded to the electric stimulation by extending new outgrowth perpendicular to the electric field with less outgrowth cathodally. [5] However, our experiments, much shorter, show no directioning. 
With respect to the stimulation time, some long term in vivo experiments are worth mentioning in relation with this work. Borgens et al. [13] applied a constant DC electric field (200 $\mu \mathrm{V} / \mathrm{mm}$ for up to 4 weeks) using steel external electrode to hemisected guinea pig spinal cords to aid regeneration, and $25 \%$ of animals exhibited improved muscle reflex in comparison with controls. These results led to a Phase 1 human clinical trial with 10 patients using the same stimulation parameters (500-600 $\mu \mathrm{V} / \mathrm{mm}, 15 \mathrm{~min}$ on / $15 \mathrm{~min}$ off, for up to 15 weeks), in which improved sensation (recovery of pinprick points) occurred in some patients. $20 \mathrm{~Hz}$ was chosen because it is the physiologically relevant frequency of motoneuron discharge. Electrical stimulation for longer than $1 \mathrm{~h}$ did not result in a further enhancement in neuronal growth, suggesting a critical stimulation time, and no further improvement afterwards. [5] This work would agree with the fact that short times below $1 \mathrm{~h}$ involve EF effects in vitro. Significantly, the results are largely dependent on the electrode material used, its charge capacity and impedance.

At present it is still an intriguing fact that cell responses (migration and directioning) depend on the cell type, the substrate used, and if the neurite becomes dendrite or axon, as mentioned in reference [2], even after considering the interrelation among chemical and electrical variables, electric gradients and ion concentration etc. In this work, no differences are observed in the extension of neurites in the parallel scars of each scratch zone, either in the positive or negative poles of the electrochemical cell. Directioning effects of the EF therefore are not significant at this cell density seeding and this short stimulation time, with respect to the neural cues that tend to fill the cell-free wound zone. It is relevant here to consider that cells may behave differently when isolated from other cells where no external signaling is detected.

Visually, the filling of the scratch empty zone seems to occur by extension of neurites starting from the edge of the scratch area and towards the opposite scar edge, no matter the side of the $\mathrm{EF}$, positive or negative. Both, in the negative and positive sides of the electrochemical cell, the two borders of the scratch are not distinguishable in neurite outgrowth. In conclusion, in terms of directioning, specific neuronal cues seem to exist that direct the filling, winning over the possible directioning EF effect. Thus, EF effects in this cell concentration and time, are seen to act in neurite regeneration but not directioning.

Finally, other electrode geometries have been tested, introducing coated platinum electrodes vertically in the cell culture, and show similar results in terms of EF effects. In this option, the absolute charge capacity may be greatly increased by increasing the surface area, while still maintaining the EF geometry for the scratch. The extension to other geometries, gives additional relevance to the in vitro model used for lesions, since it represents possible variations in implanted electrodes in vivo.

In summary, a large statistically differentiation is found among tested electroactive materials vs the uncoated Pt electrodes, where regeneration, as neurite coverage of the lesion, is greatly inhibited. Furthermore, the material is significant, since the best results come from the nanostructured IrOx-eG case with the largest CSC and lowest impedance, even with the same 
charge delivered. This aspect elicits the conclusion that any of these optimal materials improves the behavior of Platinum electrodes, and that the larger the CSC of the material, the better effect would EF stimulation have, even if only partially used. It is worth remarking the very short times, and fields at which we observed differentiating effects depending on the material used as electrode. Extended experiments in time, especially in the case of IrOx-eG, are planned, following this model of lesion which is giving clear results in electrostimulation at short times after the lesion.

\section{Conclusions}

Short term EF stimulation on in vitro scratched neuronal cell cultures as a model for a lesion evidences a clear difference in the effects of EF for the various implanted electrode materials. Factors such as nanostructure, individual chemistry and intercalation properties, charge capacity in the faradaic intercalation regime, and impedance are essential. It also shows the need to coat noble metals like Platinum with electroactive materials of large charge capacity, in order to optimize the effects of EF stimulation at low frequencies. Nanostructured IrOxgraphene and bilayer polymers with Lysine counterions have shown to prevent in great extent secondary reactions such as radical formation and allow longer time stimulations, with no harmful effects derived from ionic gradients during intercalation, and to enhance neurite outgrowth above spontaneous in the first case. Electrodes do not show a significant loss of electrochemical yield after being used in electrostimulation. Furthermore, the effects observed suggest that electrostimulation with these materials may be extended to larger frequency applications. Also, the in vitro model and EF protocols used are adequate to differentiate among materials in terms of biocompatibility in presence of fields, superior to simple cell cultures, but also to evaluate the true effects of EF application in systems where faradaic reactions at the interface have been substituted by internal electrode processes. Longer times experiments and 3D conducting systems are being explored as a natural extension of the work, as well as in vivo tests. It is also important to note that the model used is especially relevant as compared with standard cell cultures on the material, since it emulates the biological factors present in a lesion, and also allows studying regeneration in the simplest case, beyond the isolated neurons. Further extension of the model is planned in the future incorporating glial cells. 


\section{Acknowledgements}

This work was supported by grants from the Spanish MEC (MAT2011-24363 and MAT2015-65192-R), Marató TV3 Grant (110130/31), CSIC Ref. 201560 E053 and Instituto de Salud Carlos III (PI 10/453), Generalitat de Catalunya (2014/SGR/625, 2014/SGR/1643), and Severo Ochoa Programme for Centres of Excellence in R\&D (SEV- 2015-0496. L. Ballesteros thanks financing from CONICYT Fondecyt Grant № 3150143. The technical assistance of Elisenda Marti is acknowledged.

Supporting Information Material: Basic Table of materials from which a selection is made based on neural cell viability data. Figure shows the cell count after 4 DIV for each material.

\section{Conflict of interest Statement}

The authors confirm that there are no known conflicts of interest associated with this publication and there has been no significant financial support for this work that could have influenced its outcome.

Additional information: \#\# This work is the result of day by day collaboration. N. Casañ Pastor is responsible of the idea, materials, characterization, electrodes, cell design and EF application protocols, designed jointly with E. Pérez, who has synthesized the materials, coatings, characterized them. L. Ballesteros has carried out impedance measurements. C. Suñol is responsible of cell cultures and in vitro model. M. P. Lichtenstein and E. Pérez have performed jointly EF application experiments, while cell cultures along have been performed by M. P. Lichtenstein.

\section{References}

[1] C.D. McCaig, B. Song, A.M. Rajnicek, Electrical dimensions in cell science., J. Cell Sci. 122 (2009) 4267-76. doi:10.1242/jcs.023564.

[2] C.D. McCaig, A.N.N.M. Rajnicek, B. Song, M. Zhao, Controlling Cell Behavior Electrically: Current Views and Future Potential, Physiol Rev. 85 (2005) 943-978. doi:10.1152/physrev.00020.2004.

[3] J. Gardner, A history of deep brain stimulation: Technological innovation and the 
role of clinical assessment tools, Soc. Stud. Sci. 43 (2013) 707-728. doi:10.1177/0306312713483678.

[4] A.M. Kuncel, W.M. Grill, Selection of stimulus parameters for deep brain stimulation, Clin. Neurophysiol. 115 (2004) 2431-2441. doi:10.1016/j.clinph.2004.05.031.

[5] D.M. Thompson, A.N. Koppes, J.G. Hardy, C.E. Schmidt, Electrical Stimuli in the Central Nervous System Microenvironment, Annu. Rev. Biomed. Eng. 16 (2014) 397430. doi:10.1146/annurev-bioeng-121813-120655.

[6] D.R. Merrill, M. Bikson, J.G.R. Jefferys, Electrical stimulation of excitable tissue: design of efficacious and safe protocols, J. Neurosci. Methods. 141 (2005) 171-198. doi:10.1016/j.jneumeth.2004.10.020.

[7] V.S. Polikov, P.A. Tresco, W.M. Reichert, Response of brain tissue to chronically implanted neural electrodes, J. Neurosci. Methods. 148 (2005) 1-18. doi:10.1016/j.jneumeth.2005.08.015.

[8] A.M. Rajnicek, L.E. Foubister, C.D. McCaig, Alignment of corneal and lens epithelial cells by co-operative effects of substratum topography and DC electric fields, Biomaterials. 29 (2008) 2082-2095. doi:10.1016/j.biomaterials.2008.01.015.

[9] A. Golberg, M. Bei, R.L. Sheridan, M.L. Yarmush, Regeneration and control of human fibroblast cell density by intermittently delivered pulsed electric fields, Biotechnol. Bioeng. 110 (2013) 1759-1768. doi:10.1002/bit.24831.

[10] R.H.W. Funk, Endogenous electric fields as guiding cue for cell migration, Front. Physiol. 6 (2015) 1-8. doi:10.3389/fphys.2015.00143.

[11] L. Yao, A. Pandit, S. Yao, C.D. McCaig, Electric field-guided neuron migration: a novel approach in neurogenesis, Tissue Eng. Part B, Rev. 17 (2011) 143-153. doi:10.1089/ten.teb.2010.0561.

[12] K.R. Robinson, The responses of cells to electrical fields: A review, J. Cell Biol. 101 (1985) 2023-2027. doi:10.1083/jcb.101.6.2023.

[13] R.B. Borgens, Electrically mediated regeneration and guidance of adult mammalian spinal axons into polymeric channels, Neuroscience. 91 (1999) 251-264. doi:10.1016/S0306-4522(98)00584-3.

[14] S.C. Mailley, M. Hyland, P. Mailley, J.M. McLaughlin, E.T. McAdams, Electrochemical and structural characterizations of electrodeposited iridium oxide thin-film electrodes applied to neurostimulating electrical signal, Mater. Sci. Eng. C. 21 (2002) 167-175. doi:10.1016/S0928-4931(02)00098-X.

[15] S.F. Cogan, A.A. Guzelian, W.F. Agnew, T.G.H. Yuen, D.B. McCreery, Over-pulsing degrades activated iridium oxide films used for intracortical neural stimulation, J. Neurosci. Methods. 137 (2004) 141-150. doi:10.1016/j.jneumeth.2004.02.019.

[16] R.D. Meyer, S.F. Cogan, T.H. Nguyen, R.D. Rauh, Electrodeposited Iridium Oxide for Neural Stimulation and Recording Electrodes, IEEE Trans. NEURAL Syst. Rehabil. Eng. 
9 (2001) 2-11. doi:10.1109/7333.918271.

[17] A.M. Cruz, N. Casañ-Pastor, Graded conducting titanium-iridium oxide coatings for bioelectrodes in neural systems, Thin Solid Films. 534 (2013) 316-324. doi:10.1016/j.tsf.2013.02.031.

[18] A.M. Cruz, L. Abad, N.M. Carretero, J. Moral-Vico, J. Fraxedas, P. Lozano, G. Subías, V. Padial, M. Carballo, J.E. Collazos-Castro, N. Casañ-Pastor, Iridium oxohydroxide, a significant member in the family of iridium oxides. Stoichiometry, characterization, and implications in bioelectrodes, J. Phys. Chem. C. 116 (2012) 5155-5168. doi:10.1021/jp212275q.

[19] Y. Lu, T. Wang, Z. Cai, Y. Cao, H. Yang, Y.Y. Duan, Anodically electrodeposited iridium oxide films microelectrodes for neural microstimulation and recording, Sensors Actuators B Chem. 137 (2009) 334-339. doi:10.1016/j.snb.2008.11.036.

[20] S. Tsukada, H. Nakashima, K. Torimitsu, Conductive polymer combined silk fiber bundle for bioelectrical signal recording, PLoS One. 7 (2012) 1-10. doi:10.1371/journal.pone.0033689.

[21] R. Balint, N.J. Cassidy, S.H. Cartmell, Conductive polymers: Towards a smart biomaterial for tissue engineering, Acta Biomater. 10 (2014) 2341-2353. doi:10.1016/j.actbio.2014.02.015.

[22] N.K. Guimard, N. Gomez, C.E. Schmidt, Conducting polymers in biomedical engineering, Prog. Polym. Sci. $32 \quad$ (2007) 876-921. doi:10.1016/j.progpolymsci.2007.05.012.

[23] M.R. Abidian, J.M. Corey, D.R. Kipke, D.C. Martin, Conducting-polymer nanotubes improve electrical properties, mechanical adhesion, neural attachment, and neurite outgrowth of neural electrodes, Small. 6 (2010) 421-429. doi:10.1002/smll.200901868.

[24] H.C. Tian, J.Q. Liu, D.X. Wei, X.Y. Kang, C. Zhang, J.C. Du, B. Yang, X. Chen, H.Y. Zhu, Y.N. NuLi, C.S. Yang, Graphene oxide doped conducting polymer nanocomposite film for electrode-tissue interface, Biomaterials. $35 \quad$ (2014) 2120-2129. doi:10.1016/j.biomaterials.2013.11.058.

[25] H. Vara, J.E. Collazos-Castro, Biofunctionalized Conducting Polymer/Carbon Microfiber Electrodes for Ultrasensitive Neural Recordings, ACS Appl. Mater. Interfaces. 7 (2015) 27016-27026. doi:10.1021/acsami.5b09594.

[26] N.M. Carretero, M.P. Lichtenstein, E. Pérez, L. Cabana, C. Suñol, N. Casañ-Pastor, IrOx-carbon nanotube hybrids: A nanostructured material for electrodes with increased charge capacity in neural systems, Acta Biomater. 10 (2014) 4548-4558. doi:10.1016/j.actbio.2014.06.019.

[27] N.M. Carretero, M.P. Lichtenstein, E. Pérez, S. Sandoval, G. Tobias, C. Suñol, N. Casañ-Pastor, Enhanced charge capacity in iridium oxide-graphene oxide hybrids, Electrochim. Acta. 157 (2015) 369-377. doi:10.1016/j.electacta.2014.10.034. 
[28] E. Pérez, M.P. Lichtenstein, C. Suñol, N. Casañ-Pastor, Coatings of nanostructured pristine graphene-IrOx hybrids for neural electrodes: Layered stacking and the role of non-oxygenated graphene, Mater. Sci. Eng. C. 55 (2015) 218-226. doi:10.1016/j.msec.2015.05.010.

[29] S.F. Cogan, Neural stimulation and recording electrodes, Annu. Rev. Biomed. Eng. 10 (2008) 275-309. doi:10.1146/annurev.bioeng.10.061807.160518.

[30] J. Moral-Vico, N.M. Carretero, E. Pérez, C. Suñol, M. Lichtenstein, N. Casañ-Pastor, Dynamic electrodeposition of aminoacid-polypyrrole on aminoacid-PEDOT substrates: Conducting polymer bilayers as electrodes in neural systems, Electrochim. Acta. 111 (2013) 250-260. doi:10.1016/j.electacta.2013.08.041.

[31] J.E. Collazos-Castro, A.M. Cruz, M. Carballo-Vila, M. Lira-Cantú, L. Abad, Á. Pérez del Pino, J. Fraxedas, A. San Juan, C. Fonseca, A.P. Pêgo, N. Casañ-Pastor, Neural cell growth on TiO2 anatase nanostructured surfaces, Thin Solid Films. 518 (2009) 160170. doi:10.1016/j.tsf.2009.06.048.

[32] M. Carballo-Vila, B. Moreno-Burriel, E. Chinarro, J.R. Jurado, N. Casañ-Pastor, J.E. Collazos-Castro, Titanium oxide as substrate for neural cell growth, J. Biomed. Mater. Res. - Part A. 90 (2009) 94-105. doi:10.1002/jbm.a.32058.

[33] R.A. Green, N.H. Lovell, G.G. Wallace, L.A. Poole-Warren, Conducting polymers for neural interfaces: Challenges in developing an effective long-term implant, Biomaterials. 29 (2008) 3393-3399. doi:10.1016/j.biomaterials.2008.04.047.

[34] M. Baldrighi, M. Trusel, R. Tonini, S. Giordani, Carbon Nanomaterials Interfacing with Neurons: An In vivo Perspective, Front. Neurosci. 10 (2016) 1-27. doi:10.3389/fnins.2016.00250.

[35] J. Moral-Vico, S. Sánchez-Redondo, M.P. Lichtenstein, C. Suñol, N. Casañ-Pastor, Nanocomposites of iridium oxide and conducting polymers as electroactive phases in biological media, Acta Biomater. 10 (2014) 2177-2186. doi:10.1016/j.actbio.2013.12.051.

[36] J.J. Pancrazio, Neural interfaces at the nanoscale, Nanomedicine (Lond). 3 (2008) 823-830. doi:10.2217/17435889.3.6.823.

[37] M.K. Gheith, T.C. Pappas, A. V. Liopo, V.A. Sinani, B.S. Shim, M. Motamedi, J.P. Wicksted, N.A. Kotov, Stimulation of neural cells by lateral currents in conductive layer-by-layer films of single-walled carbon nanotubes, Adv. Mater. 18 (2006) 29752979. doi:10.1002/adma.200600878.

[38] A. Fabbro, D. Scaini, V. Leo, E. Vaquez, G. Cellot, G. Privitera, L. Lombardi, F. Torrisi, F. Tomarchio, F. Bonaccorso, S. Bosi, A.C. Ferrari, L. Ballerini, M. Prato, GrapheneBased Interfaces Do Not Alter Target Nerve Cells, ACS Nano. 10 (2016) 615-623. doi:10.1021/acsnano.5b05647.

[39] S.W. Hong, J.H. Lee, S.H. Kang, E.Y. Hwang, Y.S. Hwang, M.H. Lee, D.W. Han, J.C. Park, Enhanced neural cell adhesion and neurite outgrowth on graphene-based biomimetic substrates, Biomed Res. Int. (2014) 1-8. doi:10.1155/2014/212149. 
[40] A. Fabbro, A. Villari, J. Laishram, D. Scaini, F.M. Toma, A. Turco, M. Prato, L. Ballerini, Spinal cord explants use carbon nanotube interfaces to enhance neurite outgrowth and to fortify synaptic inputs, ACS Nano. 6 (2012) 2041-2055. doi:10.1021/nn203519r.

[41] V. Martinelli, G. Cellot, F.M. Toma, C.S. Long, J.H. Caldwell, L. Zentilin, M. Giacca, A. Turco, M. Prato, L. Ballerini, L. Mestroni, Carbon nanotubes promote growth and spontaneous electrical activity in cultured cardiac myocytes, Nano Lett. 12 (2012) 1831-1838. doi:10.1021/nl204064s.

[42] O. Akhavan, E. Ghaderi, S.A. Shirazian, R. Rahighi, Rolled graphene oxide foams as three-dimensional scaffolds for growth of neural fibers using electrical stimulation of stem cells, Carbon N. Y. 97 (2016) 71-77. doi:10.1016/j.carbon.2015.06.079.

[43] F. Veliev, A. Briançon-Marjollet, V. Bouchiat, C. Delacour, Impact of crystalline quality on neuronal affinity of pristine graphene, Biomaterials. 86 (2016) 33-41. doi:10.1016/j.biomaterials.2016.01.042.

[44] S. Hamid, R. Hayek, Role of electrical stimulation for rehabilitation and regeneration after spinal cord injury: An overview, Eur. Spine J. 17 (2008) 1256-1269. doi:10.1007/s00586-008-0729-3.

[45] M.P. Lichtenstein, J.L.M. Madrigal, A. Pujol, E. Galea, JNK/ERK/FAK Mediate promigratory actions of basic fibroblast growth factor in astrocytes via CCL2 and COX2, NeuroSignals. 20 (2012) 86-102. doi:10.1159/000330805.

[46] J. Moral-Vico, Materiales Electroactivos Poliméricos e Híbridos como Sustrato de Crecimiento Neuronal, 2012. https://www.educacion.gob.es/ teseo/mostrarRef.do?ref=983118/.

[47] K.K. Kasem, S. Jones, Platinum as a reference electrode in electrochemical measurements, Platin. Met. Rev. 52 (2008) 100-106. doi:10.1595/147106708X297855.

[48] A.M. Bond, P.A. Lay, Cyclic voltammetry at microelectrodes in the absence of added electrolyte using a platinum quasi-reference electrode, J. Electroanal. Chem. 199 (1986) 285-295. doi:10.1016/0022-0728(86)80004-3.

[49] H. Zhou, T. Li, Y.Y. Duan, Reduce impedance of intracortical iridium oxide microelectrodes by hydrogel coatings, Sensors Actuators, B Chem. 161 (2012) 198202. doi:10.1016/j.snb.2011.10.019.

[50] W. Franks, I. Schenker, P. Schmutz, A. Hierlemann, Impedance characterization and modeling of electrodes for biomedical applications, IEEE Trans. Biomed. Eng. 52 (2005) 1295-1302. doi:10.1109/TBME.2005.847523.

[51] W. Yang, Y. Zhao, X. He, Y. Chen, J. Xu, S. Li, Y. Yang, Y. Jiang, Flexible conducting polymer/reduced graphene oxide films: synthesis, characterization, and electrochemical performance, Nanoscale Res. Lett. 10 (2015) 1-7. doi:10.1186/s11671-015-0932-1. 
[52] J.-W. Jeong, G. Shin, S. II Park, K.J. Yu, L. Xu, J.A. Rogers, Soft materials in neuroengineering for hard problems in neuroscience, Neuron. 86 (2015) 175-186. doi:10.1016/j.neuron.2014.12.035.

[53] P. Fromherz, Electrical Interfacing of Nerve Cells and Semiconductor Chips, Chem Phys Chem. 3 (2002) 276-284. doi:10.1002/1439-7641(20020315)3:3<276::AIDCPHC276>3.0.CO;2-A.

[54] A. Arsiwala, P. Desai, V. Patravale, Recent advances in micro/nanoscale biomedical implants, J. Control. Release. 189 (2014) 25-45. doi:10.1016/j.jconrel.2014.06.021.

[55] C. Boehler, T. Stieglitz, M. Asplund, Nanostructured platinum grass enables superior impedance reduction for neural microelectrodes, Biomaterials. 67 (2015) 346-353. doi:10.1016/j.biomaterials.2015.07.036.

[56] M. Lietz, L. Dreesmann, M. Hoss, S. Oberhoffner, B. Schlosshauer, Neuro tissue engineering of glial nerve guides and the impact of different cell types, Biomaterials. 27 (2006) 1425-1436. doi:10.1016/j.biomaterials.2005.08.007.

[57] C.C. McYntire, R.W. Anderson, Deep Brain Stimulation Mechanisms: the control of neural activity via neurochemistry modulation, J. Neurochem. 139 (2016) 338-345. doi:10.1111/jnc.13649.

[58] R. Stewart, D.W. Allan, C.D. McCaig, Lectins implicate specific carbohydrate domains in electric field stimulated nerve growth and guidance, J. Neurobiol. 30 (1996) 425437. doi:10.1002/(SICI)1097-4695(199607)30:3<425::AID-NEU10>3.0.CO;2-G. 